\title{
Basic properties of a mean field laser equation
}

\author{
F. Fagnola* $\quad$ C.M. Mora ${ }^{\dagger \ddagger}$
}

\begin{abstract}
We study the non-linear quantum master equation describing a laser under the mean field approximation. The quantum system is formed by a single mode optical cavity and two level atoms, which interact with reservoirs. Namely, we establish the existence and uniqueness of the regular solution to the non-linear operator equation under consideration, as well as we get a probabilistic representation for this solution in terms of a mean field stochastic Schröndiger equation. To this end, we find a regular solution for the non-autonomous linear quantum master equation in GoriniKossakowski-Sudarshan-Lindblad form, and we prove the uniqueness of the solution to the non-autonomous linear adjoint quantum master equation in Gorini-KossakowskiSudarshan-Lindblad form. Moreover, we obtain rigorously the Maxwell-Bloch equations from the mean field laser equation.
\end{abstract}

Keywords: Open quantum system, nonlinear quantum master equation, MaxwellBloch equations, quantum master equation in the Gorini-Kossakowski-SudarshanLindblad form, existence and uniqueness, regular solution, Ehrenfest-type theorem, stochastic Schrödinger equation.

*Dipartimento di Matematica, Politecnico di Milano, Piazza Leonardo Da Vinci 32, I-20133, Milano, Italy. e-mail: franco.fagnola@polimi.it

${ }^{\dagger}$ Departamento de Ingeniería Matemática, Universidad de Concepción, Barrio Universitario, Avenida Esteban Iturra s/n, 4089100 , Casilla 160-C, Concepción, Chile. e-mail: cmora@ing-mat.udec.cl

${ }_{\ddagger}^{\ddagger}$ Partially supported by the Universidad de Concepción project VRID-Enlace 218.013.043-1.0. 


\section{Introduction}

This paper provides the mathematical foundation for the nonlinear laser equation

$$
\begin{aligned}
\frac{d}{d t} \rho_{t}= & -\mathrm{i} \frac{\omega}{2}\left[2 a^{\dagger} a+\sigma^{3}, \rho_{t}\right] \\
& +g\left[\left(\operatorname{tr}\left(\sigma^{-} \rho_{t}\right) a^{\dagger}-\operatorname{tr}\left(\sigma^{+} \rho_{t}\right) a\right)+\left(\operatorname{tr}\left(a^{\dagger} \rho_{t}\right) \sigma^{-}-\operatorname{tr}\left(a \rho_{t}\right) \sigma^{+}\right), \rho_{t}\right] \\
& +\kappa_{-}\left(\sigma^{-} \rho_{t} \sigma^{+}-\frac{1}{2} \sigma^{+} \sigma^{-} \rho_{t}-\frac{1}{2} \rho_{t} \sigma^{+} \sigma^{-}\right) \\
& +\kappa_{+}\left(\sigma^{+} \rho_{t} \sigma^{-}-\frac{1}{2} \sigma^{-} \sigma^{+} \rho_{t}-\frac{1}{2} \rho_{t} \sigma^{-} \sigma^{+}\right) \\
& +2 \kappa\left(a \rho_{t} a^{\dagger}-\frac{1}{2} a^{\dagger} a \rho_{t}-\frac{1}{2} \rho_{t} a^{\dagger} a\right),
\end{aligned}
$$

where $\omega \in \mathbb{R}, g$ is a non-zero real number, $\kappa, \kappa_{+}, \kappa_{-}>0$ and $\rho_{t}$ is an unknown nonnegative trace-class operator on $\ell^{2}\left(\mathbb{Z}_{+}\right) \otimes \mathbb{C}^{2}$. As usual, $[\cdot, \cdot]$ stands for the commutator of two operators,

$$
\sigma^{+}=\left(\begin{array}{cc}
0 & 1 \\
0 & 0
\end{array}\right), \quad \sigma^{-}=\left(\begin{array}{cc}
0 & 0 \\
1 & 0
\end{array}\right), \quad \sigma^{3}=\left(\begin{array}{cc}
1 & 0 \\
0 & -1
\end{array}\right)
$$

and $a, a^{\dagger}$ are the closed operators on $\ell^{2}\left(\mathbb{Z}_{+}\right)$given by

$$
a e_{n}= \begin{cases}\sqrt{n} e_{n-1} & \text { if } n \in \mathbb{N} \\ 0 & \text { if } n=0\end{cases}
$$

and $a^{\dagger} e_{n}=\sqrt{n+1} e_{n+1}$ for all $n \in \mathbb{Z}_{+}$. Here and subsequently, $\left(e_{n}\right)_{n \geq 0}$ denotes the canonical orthonormal basis of $\ell^{2}\left(\mathbb{Z}_{+}\right)$.

Under the mean field approximation, (1) describes the dynamics of a laser consisting of a radiation field coupled to a set of identical non-interacting two-level systems (see, e.g., Section 3.7.3 of [8] and [27, 32, 40, 47] for more details on mean field quantum master equations). The first term of the right-hand side of (1) is determined by the free Hamiltonians of the field mode and the atoms, the second term governs the atom-field interaction, and the last three terms, i.e., the Gorini-Kossakowski-Sudarshan-Lindblad superoperators [24, 31, represent decay/pumping in the atoms and radiation losses. We are interested in establishing rigorously the well-posedness of (11), the equations of motion of the observables $a+a^{\dagger}, \sigma^{-}+\sigma^{+}$and $\sigma^{3}$, and a probabilistic representation of $\rho_{t}$. This gives the mathematical basis to study, for instance, dynamical properties of (11) and the numerical solution of (11).

Our approach to the non-linear quantum master equation (1) involves the study of non-autonomous linear quantum master equations in the Gorini-Kossakowski-SudarshanLindblad (GKSL) form [1, 8, 24, 31. In the time-homogeneous setup, E. B. Davies and A. M. Chebotarev [12, 17] constructed the minimal solution of GKSL linear master equations with unbounded coefficients (see, e.g., [13, 18]). Using semigroup methods, [11, 14, 15, 18, prove that these equations have a unique solution under a quantum version of the Lyapunov condition for nonexplosion of classical Markov processes. Applying probabilistic techniques, one deduces that the GKSL quantum master equation 
preserves the regularity of the initial state (see, e.g., [37 ), and one also obtains the well-posedness of the GKSL adjoint quantum master equation with an initial condition given by an unbounded operator (see, e.g., [36]). Using a limit procedure, one gets a conservative solution to a linear adjoint quantum master equation with time-dependent coefficients (see, e.g, [10]). In this article, we address a class of time-local linear master equations, which describes relevant physical systems (see, e.g., [7, 9, 16, 26, 46]). Namely, by extending some results given by [36, 37, we construct a regular solution for the non-autonomous linear quantum master equation

$$
\frac{d}{d t} \rho_{t}=G(t) \rho_{t}+\rho_{t} G(t)^{*}+\sum_{k=1}^{\infty} L_{k}(t) \rho_{t} L_{k}(t)^{*} \quad t \geq 0,
$$

where $\rho_{t}$ is a density operator in $\mathfrak{h}$, the initial datum $\rho_{0}$ is regular, and $G(t), L_{1}(t)$, $L_{2}(t), \ldots$ are linear operators in $\mathfrak{h}$ satisfying (on appropriate domain)

$$
G(t)=-\mathrm{i} H(t)-\frac{1}{2} \sum_{\ell=1}^{\infty} L_{\ell}(t)^{*} L_{\ell}(t)
$$

with $H(t)$ self-adjoint operator in $\mathfrak{h}$. Furthermore, we prove the uniqueness of the solution to the adjoint version of (2), which models the evolution of the quantum observables in the Heisenberg picture. This leads to prove the well-posedness of the GKSL quantum master equation resulting from replacing in (1) the unknown values of $\operatorname{tr}\left(\sigma^{-} \rho_{t}\right)$ and $\operatorname{tr}\left(a \rho_{t}\right)$ by known functions $\alpha(t)$ and $\beta(t)$.

Our main objective is to develop the mathematical theory for the non-linear equation (1). First, we establish the existence and uniqueness of the regular solution to (1). In this direction, Belavkin [5, 6] treated a general class of non-linear quantum master equations with bounded coefficients, and Kolokoltsov [30] obtained the well-posedness of nonlinear quantum dynamic semigroups having non-linear Hamiltonians that are bounded perturbations of unbounded linear self-adjoint operators, together with nonlinear bounded Gorini-Kossakowski-Sudarshan-Lindblad superoperators. Arnold and Sparber [2] showed the existence and uniqueness of global solution to a non-linear quantum master equation involving Hartree potential by means of semigroup techniques.

Moreover, we deal with the equations of motion for the mean values of $a, \sigma^{-}$and $\sigma^{3}$. It is well known that the following first-order differential equations is formally obtained from (11):

$$
\left\{\begin{array}{l}
\frac{d}{d t} \operatorname{tr}\left(a \rho_{t}\right)=-(\kappa+\mathrm{i} \omega) \operatorname{tr}\left(a \rho_{t}\right)+g \operatorname{tr}\left(\sigma^{-} \rho_{t}\right) \\
\frac{d}{d t} \operatorname{tr}\left(\sigma^{-} \rho_{t}\right)=-(\gamma+\mathrm{i} \omega) \operatorname{tr}\left(\sigma^{-} \rho_{t}\right)+g \operatorname{tr}\left(a \rho_{t}\right) \operatorname{tr}\left(\sigma^{3} \rho_{t}\right) \\
\frac{d}{d t} \operatorname{tr}\left(\sigma^{3} \rho_{t}\right)=-4 g \Re\left(\operatorname{tr}\left(a \rho_{t}\right) \overline{\operatorname{tr}\left(\sigma^{-} \rho_{t}\right)}\right)-2 \gamma\left(\operatorname{tr}\left(\sigma^{3} \rho_{t}\right)-d\right)
\end{array}\right.
$$

where $\geq 0, \gamma=\left(\kappa_{+}+\kappa_{-}\right) / 2$ and $d=\left(\kappa_{+}-\kappa_{-}\right) /\left(\kappa_{+}+\kappa_{-}\right)$(see, e.g., [8]). In the semiclassical laser theory, the Maxwell-Bloch equations (3) describe the evolution of the field (i.e., $\operatorname{tr}\left(a \rho_{t}\right)$ ), the polarization (i.e., $\operatorname{tr}\left(\sigma^{-} \rho_{t}\right)$ ) and the population inversion (i.e., $\left.\operatorname{tr}\left(\sigma^{3} \rho_{t}\right)\right)$ of ring lasers like far-infrared $N H_{3}$ lasers (see, e.g., [25, 42, 48]). The system (3) has received much attention in the physical literature due to its important 
role in the description of laser dynamics (see, e.g., [8, 21, 41]). In this paper, we prove rigorously the validity of (3) whenever the initial state is regular enough, and thus we get an Ehrenfest theorem for (1) (see, e.g., [19, 22, 23]).

Finally, we obtain a probabilistic representation of (11). The solution of the linear quantum master equations in GKSL form is characterized as the mean value of random pure states given by the linear and non-linear stochastic Schrödinger equations (see, e.g., [3, 4, 8, 37, 49]). This representation plays an important tool in the numerical simulation of open quantum systems (see, e.g., [8, 35, 33, 43, 45]), and it has also been used for proving theoretical properties of the GKSL quantum master equations (see, e.g., [20, 36, 37]). In this paper, we get a probabilistic representation of (11) in terms of a mean field version of the linear stochastic Schrödinger equation. To the best of our knowledge this is the first rigorously established result, at the level of infinite dimensional density matrices, with an unbounded nonlinear evolution operator, in the study of nonlinear mean field laser evolution equations

This paper is organized as follows. Section 2 presents the main results. Section 3 is devoted to general linear master equations. In Section 4 we study a linear quantum master equation associated with (11), moreover, for the sake of completeness, we recall the basic properties of the complex Lorenz equations. All proofs are deferred to Section 5.

\section{$1.1 \quad$ Notation}

In this paper, $(\mathfrak{h},\langle\cdot, \cdot\rangle)$ is a separable complex Hilbert space, where the scalar product $\langle\cdot, \cdot\rangle$ is linear in the second variable and anti-linear in the first one. The standard basis

of $\mathbb{C}^{2}$ is denoted by $e_{+}=\left(\begin{array}{l}1 \\ 0\end{array}\right)$ and $e_{-}=\left(\begin{array}{l}0 \\ 1\end{array}\right)$. If $A, B$ are linear operators in $\mathfrak{h}$, then $[A, B]=A B-B A$ and $\mathcal{D}(A)$ stands for the domain of $A$. We take $N=a^{\dagger} a$. In case $\mathfrak{X}, \mathfrak{Z}$ are normed spaces, we denote by $\mathfrak{L}(\mathfrak{X}, \mathfrak{Z})$ the set of all bounded operators from $\mathfrak{X}$ to $\mathfrak{Z}$ and we choose $\mathfrak{L}(\mathfrak{X})=\mathfrak{L}(\mathfrak{X}, \mathfrak{X})$. We write $\mathfrak{L}_{1}(\mathfrak{h})$ for the set of all trace-class operators on $\mathfrak{h}$ equipped with the trace norm. For simplicity of notation, generic no-negative constants are denoted by $K$, as well as $K(\cdot)$ stands for different non-decreasing non-negative functions on $[0, \infty[$.

Let $C$ be a self-adjoint positive operator in $\mathfrak{h}$. Then, $\pi_{C}: \mathfrak{h} \rightarrow \mathfrak{h}$ is defined by $\pi_{C}(x)=x$ if $x \in \mathcal{D}(C)$ and $\pi_{C}(x)=0$ if $x \notin \mathcal{D}(C)$, as well as $\|x\|_{C}=\sqrt{\langle x, x\rangle_{C}}$ with $\langle x, y\rangle_{C}=\langle x, y\rangle+\langle C x, C y\rangle$ for any $x, y \in \mathcal{D}(C)$. We write $L^{2}(\mathbb{P}, \mathfrak{h})$ for the set of all square integrable random variables from $(\Omega, \mathfrak{F}, \mathbb{P})$ to $(\mathfrak{h}, \mathfrak{B}(\mathfrak{h}))$, where $\mathcal{B}(\mathfrak{Y})$ is the collection of all Borel set of the topological space $\mathfrak{Y}$. Finally, $L_{C}^{2}(\mathbb{P}, \mathfrak{h})$ denotes the set of all $\xi \in L^{2}(\mathbb{P}, \mathfrak{h})$ satisfying $\xi \in \mathcal{D}(C)$ a.s. and $\mathbb{E}\left(\|\xi\|_{C}^{2}\right)<\infty$.

\section{Basic properties of the mean field laser equa- tion}

This section presents the main results of the paper, which are summarized in Theorem 2.1 given below. We start by adapting the notion of regular weak solution - of a linear 
quantum master equation (see, e.g., 37] and Definition 3.2 given below) - to the mean field laser equation (11). To this end, we recall that a density operator $\varrho$ is $C$-regular if, roughly speaking, $C \varrho C$ is a trace-class operator, where $C$ is a suitable reference operator (see, e.g., [11, 37]).

Definition 2.1. Suppose that $C$ is a self-adjoint positive operator in $\mathfrak{h}$. An operator $\varrho \in \mathfrak{L}_{1}(\mathfrak{h})$ is called density operator iff $\varrho$ is a non-negative operator with unit trace. The non-negative operator $\varrho \in \mathfrak{L}(\mathfrak{h})$ is said to be C-regular iff $\varrho=\sum_{n \in \mathfrak{I}} \lambda_{n}\left|u_{n}\right\rangle\left\langle u_{n}\right|$ for some countable set $\mathfrak{I}$, summable non-negative real numbers $\left(\lambda_{n}\right)_{n \in \mathfrak{I}}$ and collection $\left(u_{n}\right)_{n \in \mathfrak{I}}$ of elements of $\mathcal{D}(C)$, which together satisfy: $\sum_{n \in \mathfrak{I}} \lambda_{n}\left\|C u_{n}\right\|^{2}<\infty$. Let $\mathfrak{L}_{1, C}^{+}(\mathfrak{h})$ denote the set of all $C$-regular density operators in $\mathfrak{h}$.

Definition 2.2. Let $C$ be a self-adjoint positive operator in $\mathfrak{h}$. A family $\left(\rho_{t}\right)_{t \geq 0}$ of operators belonging to $\mathfrak{L}_{1, C}^{+}(\mathfrak{h})$ is called $C$-weak solution to (1) iff the function $t \mapsto$ $\operatorname{tr}\left(a \rho_{t}\right)$ is continuous and for all $t \geq 0$ we have

$$
\frac{d}{d t} \operatorname{tr}\left(A \rho_{t}\right)=\operatorname{tr}\left(A \mathcal{L}_{\star}\left(\rho_{t}\right) \rho_{t}\right) \quad \forall A \in \mathfrak{L}(\mathfrak{h}),
$$

where

$$
\begin{aligned}
\mathcal{L}_{\star}(\widetilde{\varrho}) \varrho= & -\frac{\mathrm{i} \omega}{2}\left[2 a^{\dagger} a+\sigma^{3}, \varrho\right]+2 \kappa\left(a \varrho a^{\dagger}-\frac{1}{2} a^{\dagger} a \varrho-\frac{1}{2} \varrho a^{\dagger} a\right) \\
& +\kappa_{-}\left(\sigma^{-} \varrho \sigma^{+}-\frac{1}{2} \sigma^{+} \sigma^{-} \varrho-\frac{1}{2} \varrho \sigma^{+} \sigma^{-}\right) \\
& +\kappa_{+}\left(\sigma^{+} \varrho \sigma^{-}-\frac{1}{2} \sigma^{-} \sigma^{+} \varrho-\frac{1}{2} \varrho \sigma^{-} \sigma^{+}\right) \\
& +g\left[\operatorname{tr}\left(\sigma^{-} \widetilde{\varrho}\right) a^{\dagger}-\operatorname{tr}\left(\sigma^{+} \widetilde{\varrho}\right) a, \varrho\right]+g\left[\operatorname{tr}\left(a^{\dagger} \widetilde{\varrho}\right) \sigma^{-}-\operatorname{tr}(a \varrho) \sigma^{+}, \varrho\right] .
\end{aligned}
$$

Similar to the linear case, (1) is strongly related with the following non-linear stochastic evolution equation on $\ell^{2}\left(\mathbb{Z}_{+}\right) \otimes \mathbb{C}^{2}$ :

$$
\begin{aligned}
Z_{t}(\xi)= & \xi+\int_{0}^{t}\left(-\mathrm{i} H\left(t, Z_{t}(\xi)\right)-\frac{1}{2} \sum_{\ell=1}^{3} L_{\ell}^{*} L_{\ell}\right) Z_{s}(\xi) d s \\
& +\sum_{\ell=1}^{3} \int_{0}^{t} L_{\ell} Z_{s}(\xi) d W_{s}^{\ell}
\end{aligned}
$$

where $L_{1}=\sqrt{2 \kappa} a, L_{2}=\sqrt{\gamma(1-d)} \sigma^{-}, L_{3}=\sqrt{\gamma(1+d)} \sigma^{+}$,

$$
\begin{aligned}
H\left(t, Z_{t}(\xi)\right)= & \frac{\omega}{2}\left(2 a^{\dagger} a+\sigma^{3}\right) \\
& +\mathrm{i} g\left(\mathbb{E}\left\langle Z_{t}(\xi), \sigma^{-} Z_{t}(\xi)\right\rangle a^{\dagger}-\mathbb{E}\left\langle Z_{t}(\xi), \sigma^{+} Z_{t}(\xi)\right\rangle a\right) \\
& +\mathrm{i} g\left(\mathbb{E}\left\langle Z_{t}(\xi), a^{\dagger} Z_{t}(\xi)\right\rangle \sigma^{-}-\mathbb{E}\left\langle Z_{t}(\xi), a Z_{t}(\xi)\right\rangle \sigma^{+}\right),
\end{aligned}
$$

and $W^{1}, W^{2}, W^{3}$ are real valued independent Wiener processes on a filtered complete probability space $\left(\Omega, \mathfrak{F},\left(\mathfrak{F}_{t}\right)_{t \geq 0}, \mathbb{P}\right)$. Next, we tailor the concept of regular weak solution —used in the framework of stochastic Schödinger equations (see, e.g., [19, 38, 39] and Definition 3.1 given below) - to suit (4). 
Definition 2.3. Let $p \in \mathbb{N}$. An $\ell^{2}\left(\mathbb{Z}_{+}\right) \otimes \mathbb{C}^{2}$-valued adapted process with continuous sample paths $\left(Z_{t}(\xi)\right)_{t \in \mathbb{I}}$ is called strong $N^{p}$-solution of (4) if:

- For all $t \geq 0: \mathbb{E}\left\|Z_{t}(\xi)\right\|^{2} \leq K(t) \mathbb{E}\|\xi\|^{2}, Z_{t}(\xi) \in \mathcal{D}\left(N^{p}\right)$ a.s., and

$$
\sup _{s \in[0, t]} \mathbb{E}\left\|N^{p} X_{s}(\xi)\right\|^{2}<\infty .
$$

- The functions $t \mapsto \mathbb{E}\left\langle Z_{t}(\xi), \sigma^{-} Z_{t}(\xi)\right\rangle$ and $t \mapsto \mathbb{E}\left\langle Z_{t}(\xi)\right.$, a $\left.Z_{t}(\xi)\right\rangle$ are continuous.

- a.s. for all $t \geq 0$ :

$$
\begin{aligned}
Z_{t}(\xi)= & \xi+\int_{0}^{t}\left(-\mathrm{i} H(t)-\frac{1}{2} \sum_{\ell=1}^{3} L_{\ell}^{*} L_{\ell}\right) \pi_{N^{p}}\left(Z_{s}(\xi)\right) d s \\
& +\sum_{\ell=1}^{3} \int_{0}^{t} L_{\ell} \pi_{N^{p}}\left(Z_{s}(\xi)\right) d W_{s}^{\ell}
\end{aligned}
$$

with $H\left(t, Z_{t}(\xi)\right)$ described by (5), and $L_{\ell}, W^{\ell}$ as in (4).

Now, we establish the existence and uniqueness of the regular solution to (1), a Ehrenfest-type theorem describing the evolution of the mean values of the observables $a+a^{\dagger}, \sigma^{-}+\sigma^{+}$and $\sigma^{3}$, and the probabilistic representation of (1).

Theorem 2.1. Suppose that $\varrho \in \mathfrak{L}_{1, N^{p}}^{+}\left(\ell^{2}\left(\mathbb{Z}_{+}\right) \otimes \mathbb{C}^{2}\right)$, with $p \in \mathbb{N}$. Then, there exists a unique $N^{p}$-weak solution $\left(\rho_{t}\right)_{t \geq 0}$ to (1) with initial datum $\varrho$. Moreover, the MaxwellBloch equations (3) hold, and

$$
\rho_{t}=\mathbb{E}\left|Z_{t}(\xi)\right\rangle\left\langle Z_{t}(\xi)\right| \quad \forall t \geq 0
$$

where $\xi \in L_{N^{p}}^{2}(\mathbb{P}, \mathfrak{h})$ satisfies $\varrho=\mathbb{E}|\xi\rangle\langle\xi|$, and $Z_{t}(\xi) \in \ell^{2}\left(\mathbb{Z}_{+}\right) \otimes \mathbb{C}^{2}$ is the strong $N^{p}$-solution of (4).

Proof. Deferred to Section 5.3 .

Remark 2.1. If $g^{2} d<\kappa \gamma$, then $(0,0, d)$ is an asymptotically stable equilibrium point of (3). In fact, from (23) and (24), given below, it follows that $\operatorname{tr}\left(a \rho_{t}\right), \operatorname{tr}\left(\sigma^{-} \rho_{t}\right)$ and $\operatorname{tr}\left(\sigma^{3} \rho_{t}\right)-d$ converge exponentially fast to 0 as $t$ goes to $+\infty$.

\section{General linear quantum master equations}

\subsection{Regular solution for the GKSL quantum master equa- tion}

This subsection provides a regular solution for the linear quantum master equation (2). By generalizing [37] to the non-autonomous framework, we will describe a solution of (2) with the help of the linear stochastic evolution equation in $\mathfrak{h}$ :

$$
X_{t}(\xi)=\xi+\int_{0}^{t} G(s) X_{s}(\xi) d s+\sum_{\ell=1}^{\infty} \int_{0}^{t} L_{\ell}(s) X_{s}(\xi) d W_{s}^{\ell}
$$


where $W^{1}, W^{2}, \ldots$ are real valued independent Wiener processes on a filtered complete probability space $\left(\Omega, \mathfrak{F},\left(\mathfrak{F}_{t}\right)_{t \geq 0}, \mathbb{P}\right)$.

Suppose that the density operator $\rho_{0}$ is $C$-regular. According to Theorem 3.1 of [37] we have $\rho_{0}=\mathbb{E}|\xi\rangle\langle\xi|$ for certain $\xi \in L_{C}^{2}(\mathbb{P}, \mathfrak{h})$. We set

$$
\rho_{t}:=\mathbb{E}\left|X_{t}(\xi)\right\rangle\left\langle X_{t}(\xi)\right|
$$

where we use Dirac notation, $X_{t}(\xi)$ is the unique strong $C$-solution of (7) (see Definition 3.1), and the mathematical expectation can be interpreted as a Bochner integral in both $\mathfrak{L}_{1}(\mathfrak{h})$ and $\mathfrak{L}(\mathfrak{h})$. Then, $\rho_{t}$ is a $C$-regular density operator (see [37] for details).

Hypothesis 1. There exists a self-adjoint positive operator $C$ in $\mathfrak{h}$ such that $\mathcal{D}(C) \subset$ $\mathcal{D}(G(t))$ and $\mathcal{D}(C) \subset \mathcal{D}\left(L_{\ell}(t)\right)$ for all $t \geq 0$, and $G(\cdot) \circ \pi_{C}$ and $L_{\ell}(\cdot) \circ \pi_{C}$ are measurable as functions from $([0, \infty[\times \mathfrak{h}, \mathcal{B}([0, \infty[\times \mathfrak{h}))$ to $(\mathfrak{h}, \mathcal{B}(\mathfrak{h}))$.

Definition 3.1. Assume Hypothesis 1, Let $\mathbb{I}$ be either $\left[0, \infty\left[\right.\right.$ or $[0, T]$, with $T \in \mathbb{R}_{+}$. By strong $C$-solution of (7) with initial condition $\xi$, on the interval $\mathbb{I}$, we mean an $\mathfrak{h}$ valued adapted process $\left(X_{t}(\xi)\right)_{t \in \mathbb{I}}$ with continuous sample paths such that for all $t \in \mathbb{I}$ : $\mathbb{E}\left\|X_{t}(\xi)\right\|^{2} \leq K(t) \mathbb{E}\|\xi\|^{2}, X_{t}(\xi) \in \mathcal{D}(C)$ a.s., $\sup _{s \in[0, t]} \mathbb{E}\left\|C X_{s}(\xi)\right\|^{2}<\infty$, and

$$
X_{t}(\xi)=\xi+\int_{0}^{t} G(s) \pi_{C}\left(X_{s}(\xi)\right) d s+\sum_{\ell=1}^{\infty} \int_{0}^{t} L_{\ell}(s) \pi_{C}\left(X_{s}(\xi)\right) d W_{s}^{\ell} \quad \text { a.s. }
$$

The following theorem, which extends Theorem 4.4 of [37] to the non-autonomous context, asserts that $\rho_{t}$ given by (8) is a regular solution to (2).

Definition 3.2. Let $C$ be a self-adjoint positive operator in $\mathfrak{h}$. A family $\left(\rho_{t}\right)_{t \geq 0}$ of $C$-regular density operators is called $C$-weak solution to (2) if and only if

$$
\frac{d}{d t} \operatorname{tr}\left(A \rho_{t}\right)=\operatorname{tr}\left(A\left(G(t) \rho_{t}+\rho_{t} G(t)^{*}+\sum_{\ell=1}^{\infty} L_{\ell}(t) \rho_{t} L_{\ell}(t)^{*}\right)\right)
$$

for all $A \in \mathfrak{L}(\mathfrak{h})$ and $t \geq 0$.

Hypothesis 2. Suppose that $C$ satisfies Hypothesis 1, together with:

(H2.1) For any $t \geq 0$ and $x \in \mathcal{D}(C),\|G(t) x\|^{2} \leq K(t)\|x\|_{C}^{2}$.

(H2.2) For any $t \geq 0$ and $x \in \mathcal{D}(C), 2 \Re\langle x, G(t) x\rangle+\sum_{\ell=1}^{\infty}\left\|L_{\ell}(t) x\right\|^{2}=0$.

(H2.3) For any initial datum $\xi \in L_{C}^{2}(\mathbb{P}, \mathfrak{h})$, (7) has a unique strong $C$-solution on any bounded interval.

(H2.4) There exist functions $f_{k}:\left[0, \infty\left[\times\left[0, \infty\left[\rightarrow\left[0, \infty\left[\right.\right.\right.\right.\right.\right.$ such that: (i) $f_{k}$ is bounded on bounded subintervals of $\left[0, \infty\left[\times\left[0, \infty\left[\right.\right.\right.\right.$; (ii) $\lim _{s \rightarrow t} f_{k}(s, t)=0$; and (iii) for all $s, t \geq 0$ and $x \in \mathcal{D}(C)$ we have $\|G(s) x-G(t) x\|^{2} \leq f_{0}(s, t)\|x\|_{C}^{2}$ and $\left\|L_{\ell}(s) x-L_{\ell}(t) x\right\|^{2} \leq f_{\ell}(s, t)\|x\|_{C}^{2}$. 
Theorem 3.1. Let Hypotheses 1 and 0 hold. Assume that $\varrho_{0}$ be $C$-regular, and that $G(t), L_{1}(t), L_{2}(t), \ldots$ are closable for all $t \geq 0$. Then $\rho_{t}$ given by (8) is a $C$-weak solution to (2). Moreover, for all $t \geq 0$ we have

$$
\rho_{t}=\rho_{0}+\int_{0}^{t}\left(G(s) \rho_{s}+\rho_{s} G(s)^{*}+\sum_{\ell=1}^{\infty} L_{\ell}(s) \rho_{s} L_{\ell}(s)^{*}\right) d s,
$$

where we understand the above integral in the sense of Bochner integral in $\mathfrak{L}_{1}(\mathfrak{h})$.

Proof. Deferred to Section 5.1.1.

Remark 3.1. Sufficient conditions for the regularity of the solution to the linear stochastic Schödinger equation (7) (i.e., Hypothesis 2.3) are given, for instance, in [19, 34, 38].

\subsection{Uniqueness of the solution to the adjoint quantum master equation in the GKSL form}

The next theorem introduces the operator $\mathcal{T}_{t}(A)$ that describes the evolution of the observable $A$ at time $t$ in the Heisenberg picture. Roughly speaking, the maps $A \mapsto$ $\mathcal{T}_{t}(A)$ is the adjoint operator of the application $\varrho \mapsto \rho_{t}$, where $\rho_{t}$ is defined by (8)).

Hypothesis 3. Let Hypothesis 1 hold, together with Conditions H2.1 and H2.3. Suppose that

(H3.1) For all $t \geq 0$ and $x \in \mathcal{D}(C)$,

$$
2 \Re\langle x, G(t) x\rangle+\sum_{\ell=1}^{\infty}\left\|L_{\ell}(t) x\right\|^{2} \leq K(t)\|x\|^{2} .
$$

Theorem 3.2. Assume that Hypothesis 1 and Conditions H2.1 and H2.3 holds. Consider $A \in \mathfrak{L}(\mathfrak{h})$. Then, for every $t \geq 0$ there exists a unique $\mathcal{T}_{t}(A) \in \mathfrak{L}(\mathfrak{h})$ for which:

$$
\left\langle x, \mathcal{T}_{t}(A) y\right\rangle=\mathbb{E}\left\langle X_{t}(x), A X_{t}(y)\right\rangle \quad \forall x, y \in \mathcal{D}(C) .
$$

Moreover, $\sup _{t \in[0, T]}\left\|\mathcal{T}_{t}(A)\right\|<\infty$ for all $T \geq 0$.

Proof. Deferred to Section 5.1.2.

Theorem 3.3 below shows that $\mathcal{T}_{t}(A)$ is the unique possible solution of the adjoint quantum master equation

$$
\frac{d}{d t} \mathcal{T}_{t}(A)=\mathcal{T}_{t}(A) G(t)+G(t)^{*} \mathcal{T}_{t}(A)+\sum_{k=1}^{\infty} L_{k}(t)^{*} \mathcal{T}_{t}(A) L_{k}(t)
$$

Thus, we generalize Theorem 2.2 of [37] to the non-autonomous framework. 
Theorem 3.3. Let Hypothesis 3 hold, and let $\mathcal{T}_{t}(A)$ be as in Theorem 3.2 with $A \in$ $\mathfrak{L}(\mathfrak{h})$. Assume that $\left(\mathcal{A}_{t}\right)_{t \geq 0}$ is a family of operators belonging to $\mathfrak{L}(\mathfrak{h})$ such that $\mathcal{A}_{0}=A$, $\sup _{s \in[0, t]}\left\|\mathcal{A}_{s}\right\|_{\mathfrak{L}(\mathfrak{h})}<\infty$, and

$$
\frac{d}{d t}\left\langle x, \mathcal{A}_{t} y\right\rangle=\left\langle x, \mathcal{A}_{t} G(t) y\right\rangle+\left\langle G(t) x, \mathcal{A}_{t} y\right\rangle+\sum_{\ell=1}^{\infty}\left\langle L_{\ell}(t) x, \mathcal{A}_{t} L_{\ell}(t) y\right\rangle
$$

for all $x, y \in \mathcal{D}(C)$. Then $\mathcal{A}_{t}=\mathcal{T}_{t}(A)$ for all $t \geq 0$.

Proof. Deferred to Section 5.1.3.

Remark 3.2. In the autonomous case, [36, 37] obtain sufficient conditions for $\mathcal{T}_{t}(A)$ defined by (11) to be solution of (12). Using semigroup methods, [11, 14, 15, 18] show the existence and uniqueness of solutions to (2) and (12), in the semigroup sense.

In order to check Condition H2.3 we establish the following extension of Theorem 2.4 of [19].

Hypothesis 4. Suppose that $C$ satisfies Hypothesis 1, together with:

(H4.1) For any $t \geq 0$ and $x \in \mathcal{D}(C),\|G(t) x\|^{2} \leq K(t)\|x\|_{C}^{2}$.

(H4.2) For every $\ell \in \mathbb{N}$ there exists a non-decreasing function $K_{\ell}:[0, \infty[\rightarrow[0, \infty[$ satisfying $\left\|L_{\ell}(t) x\right\|^{2} \leq K_{\ell}(t)\|x\|_{C}^{2}$ for all $x \in \mathcal{D}(C)$ and $t \geq 0$.

(H4.3) There exists a non-decreasing function $\alpha:\left[0, \infty\left[\rightarrow\left[0, \infty\left[\right.\right.\right.\right.$ and a core $\mathfrak{D}_{1}$ of $C^{2}$ such that for any $x \in \mathfrak{D}_{1}$ we have

$$
2 \Re\left\langle C^{2} x, G(t) x\right\rangle+\sum_{\ell=1}^{\infty}\left\|C L_{\ell}(t) x\right\|^{2} \leq \alpha(t)\|x\|_{C}^{2} \quad \forall t \geq 0 .
$$

(H4.4) There exists a non-decreasing function $\beta:\left[0, \infty\left[\rightarrow\left[0, \infty\left[\right.\right.\right.\right.$ and a core $\mathfrak{D}_{2}$ of $C$ such that

$$
2 \Re\langle x, G(t) x\rangle+\sum_{\ell=1}^{\infty}\left\|L_{\ell}(t) x\right\|^{2} \leq \beta(t)\|x\|^{2} \quad \forall t \geq 0 \text { and } \forall x \in \mathfrak{D}_{2} .
$$

Theorem 3.4. In addition to Hypothesis 4 , we assume that $\xi \in L_{C}^{2}(\mathbb{P}, \mathfrak{h})$ is $\mathfrak{F}_{0}$-measurable. Then (7) has a unique strong $C$-solution $\left(X_{t}(\xi)\right)_{t \geq 0}$ with initial condition $\xi$. Moreover,

$$
\mathbb{E}\left\|C X_{t}(\xi)\right\|^{2} \leq K(t)\left(\mathbb{E}\|C \xi\|^{2}+\mathbb{E}\|\xi\|^{2}\right) .
$$

Proof. Our assertion can be be proved in much the same way as Theorem 2.4 of [19].

Remark 3.3. Theorem 3.4 given above asserts that Theorem 2.4 of [19] still holds if we replace the assumption H2.4 of [19] by Hypothesis H4.4. We will apply Theorem 3.3 to the case: $L_{1}=\sqrt{2 \kappa} a^{\dagger}, L_{2}=\sqrt{\gamma(1-d)} \sigma^{+}, L_{3}=\sqrt{\gamma(1+d)} \sigma^{-}$and $G(t)=$ $\mathrm{i} H(t)-\frac{1}{2} \sum_{\ell=1}^{3} L_{\ell} L_{\ell}^{*}$ with

$$
H(t)=\frac{\omega}{2}\left(2 a^{\dagger} a+\sigma^{3}\right)+\mathrm{i} g\left(\alpha(t) a^{\dagger}-\overline{\alpha(t)} a\right)+\mathrm{i} g\left(\overline{\beta(t)} \sigma^{-}-\beta(t) \sigma^{+}\right) .
$$

Since $G(t)+G(t)^{*}+\sum_{\ell=1}^{3} L_{\ell}^{*} L_{\ell}=4 \kappa^{2} I+2 \gamma^{2}\left(1+d^{2}\right) \sigma_{3}$, Condition H2.4 of Theorem 2.4 of [19] does not apply to our situation. 


\section{Auxiliary equations}

\subsection{Auxiliary linear quantum master equation}

This subsection deals with the linear evolution equation obtained by replacing in (11) the unknown functions $t \mapsto g \operatorname{tr}\left(\sigma^{-} \rho_{t}\right)$ and $t \mapsto g \operatorname{tr}\left(a \rho_{t}\right)$ by general functions $\alpha, \beta$ : $[0, \infty[\rightarrow \mathbb{C}$. More precisely, we study the well-posedness of the linear quantum master equation

$$
\frac{d}{d t} \rho_{t}=\mathcal{L}_{\star}^{h} \rho_{t}+\left[\alpha(t) a^{\dagger}-\overline{\alpha(t)} a+\overline{\beta(t)} \sigma^{-}-\beta(t) \sigma^{+}, \rho_{t}\right],
$$

where $\rho_{t} \in \mathfrak{L}_{1}^{+}\left(\ell^{2}\left(\mathbb{Z}_{+}\right) \otimes \mathbb{C}^{2}\right)$,

$$
\begin{aligned}
\mathcal{L}_{\star}^{h} \varrho= & {\left[-\frac{\mathrm{i} \omega}{2}\left(2 a^{\dagger} a+\sigma^{3}\right), \varrho\right]+2 \kappa\left(a \varrho a^{\dagger}-\frac{1}{2} a^{\dagger} a \varrho-\frac{1}{2} \varrho a^{\dagger} a\right) } \\
& +\gamma(1-d)\left(\sigma^{-} \varrho \sigma^{+}-\frac{1}{2} \sigma^{+} \sigma^{-} \varrho-\frac{1}{2} \varrho \sigma^{+} \sigma^{-}\right) \\
& +\gamma(1+d)\left(\sigma^{+} \varrho \sigma^{-}-\frac{1}{2} \sigma^{-} \sigma^{+} \varrho-\frac{1}{2} \varrho \sigma^{-} \sigma^{+}\right),
\end{aligned}
$$

$d \in]-1,1[, \omega \in \mathbb{R}$ and $\kappa, \gamma>0$. Furthermore, we represent (14) by using

$$
X_{t}(\xi)=\xi+\int_{0}^{t} G(s) X_{s}(\xi) d s+\sum_{\ell=1}^{3} \int_{0}^{t} L_{\ell}(s) X_{s}(\xi) d W_{s}^{\ell},
$$

where $X_{t}(\xi) \in \ell^{2}\left(\mathbb{Z}_{+}\right) \otimes \mathbb{C}^{2}, W^{1}, W^{2}, W^{3}$ are real valued independent Wiener processes, $L_{1}=\sqrt{2 \kappa} a, L_{2}=\sqrt{\gamma(1-d)} \sigma^{-}, L_{3}=\sqrt{\gamma(1+d)} \sigma^{+}$and $G(t)=-\mathrm{i} H(t)-$ $\frac{1}{2} \sum_{\ell=1}^{3} L_{\ell}^{*} L_{\ell}$ with

$$
H(t)=\frac{\omega}{2}\left(2 a^{\dagger} a+\sigma^{3}\right)+\mathrm{i}\left(\alpha(t) a^{\dagger}-\overline{\alpha(t)} a\right)+\mathrm{i}\left(\overline{\beta(t)} \sigma^{-}-\beta(t) \sigma^{+}\right) .
$$

Though the open quantum system (14) deserves attention in its own right, our main objective is to develop key tools for proving the results of Section 2, First, combining Theorems 3.1 , 3.3 and 3.4 we obtain the existence and uniqueness of the regular solution to (14).

Theorem 4.1. Consider (14) with $\alpha, \beta:\left[0, \infty\left[\rightarrow \mathbb{C}\right.\right.$ continuous. Let $\varrho$ be $N^{p}$-regular, where $p \in \mathbb{N}$. Then, there exists a unique $N^{p}$-weak solution $\left(\rho_{t}\right)_{t \geq 0}$ to (14) with initial datum $\rho_{0}=\varrho$. Moreover, for any $t \geq 0$ we have

$$
\rho_{t}=\rho_{0}+\int_{0}^{t}\left(\mathcal{L}_{\star}^{h} \rho_{s}+\left[\alpha(s) a^{\dagger}-\overline{\alpha(s)} a+\overline{\beta(s)} \sigma^{-}-\beta(s) \sigma^{+}, \rho_{s}\right]\right) d s
$$

and

$$
\rho_{t}=\mathbb{E}\left|X_{t}(\xi)\right\rangle\left\langle X_{t}(\xi)\right| \quad \forall t \geq 0,
$$

where the integral appearing in (17) is understood in the sense of Bochner integral in $\mathfrak{L}_{1}\left(\ell^{2}\left(\mathbb{Z}_{+}\right) \otimes \mathbb{C}^{2}\right), \xi \in L_{N^{p}}^{2}\left(\mathbb{P}, \ell^{2}\left(\mathbb{Z}_{+}\right) \otimes \mathbb{C}^{2}\right)$ satisfies $\varrho=\mathbb{E}|\xi\rangle\langle\xi|$ and $X_{t}(\xi)$ is the unique strong $N^{p}$-solution of (16). 
Proof. Deferred to Section 5.2.1.

Remark 4.1. Assume the framework of Theorem 4.1. From the proof of Theorem 4.1. it follows that $\mathbb{E}\left\|X_{t}(\xi)\right\|_{N^{p}}^{2} \leq K(t) \mathbb{E}\|\xi\|_{N^{p}}^{2}$ for all $t \geq 0$. In the operator language we have $\operatorname{tr}\left(N^{p} \rho_{t} N^{p}\right) \leq K(t)\left(1+\operatorname{tr}\left(N^{p} \rho_{0} N^{p}\right)\right)$ (see, e.g., [37]) since $\mathbb{E}\left\|X_{t}(\xi)\right\|^{2}=$ $\mathbb{E}\|\xi\|^{2}=1$ (see, e.g., [19]).

Using the Ehrenfest-type theorem given in [19] we get a system of ordinary differential equations that describes the evolution of $\operatorname{tr}\left(\rho_{t} a\right), \operatorname{tr}\left(\rho_{t} \sigma^{-}\right)$and $\operatorname{tr}\left(\rho_{t} \sigma^{3}\right)$.

Theorem 4.2. Under the assumptions and notation of Theorem 4.1.

$$
\begin{aligned}
\frac{d}{d t} \operatorname{tr}\left(\rho_{t} a\right)= & -(\kappa+\mathrm{i} \omega) \operatorname{tr}\left(\rho_{t} a\right)+\alpha(t), \\
\frac{d}{d t} \operatorname{tr}\left(\rho_{t} \sigma^{-}\right)= & -(\gamma+\mathrm{i} \omega) \operatorname{tr}\left(\rho_{t} \sigma^{-}\right)+\beta(t) \operatorname{tr}\left(\rho_{t} \sigma^{3}\right), \\
\frac{d}{d t} \operatorname{tr}\left(\rho_{t} \sigma^{3}\right)= & -2\left(\overline{\beta(t)} \operatorname{tr}\left(\rho_{t} \sigma^{-}\right)+\beta(t) \overline{\operatorname{tr}\left(\rho_{t} \sigma^{-}\right)}\right) \\
& -2 \gamma\left(\operatorname{tr}\left(\rho_{t} \sigma^{3}\right)-d\right) .
\end{aligned}
$$

Proof. Deferred to Section 5.2.2.

\subsection{Complex Lorenz equations}

Taking $A(t)=\operatorname{tr}\left(a \rho_{t}\right), S(t)=\operatorname{tr}\left(\sigma^{-} \rho_{t}\right)$ and $D(t)=\operatorname{tr}\left(\sigma^{3} \rho_{t}\right)$ we rewrite (3) as

$$
\left\{\begin{array}{l}
\frac{d}{d t} A(t)=-(\kappa+\mathrm{i} \omega) A(t)+g S(t) \\
\frac{d}{d t} S(t)=-(\gamma+\mathrm{i} \omega) S(t)+g A(t) D(t) \\
\frac{d}{d t} D(t)=-4 g \Re(\overline{A(t)} S(t))-2 \gamma(D(t)-d)
\end{array}\right.
$$

where $t \geq 0, D(t) \in \mathbb{R}$ and $A(t), Y(t) \in \mathbb{C}$. The complex Lorenz equation (22) has received much attention in the physical literature (see, e.g., [21, 41]) due to its important role in the description of laser dynamics. Just for the sake of completeness, we next present relevant properties of (22), together with their mathematical proofs.

Theorem 4.3. Suppose that $d \in]-1,1[, \omega \in \mathbb{R}, g \in \mathbb{R} \backslash\{0\}$ and $\kappa, \gamma>0$. Then, for every initial condition $A(0) \in \mathbb{C}, S(0) \in \mathbb{C}, D(0) \in \mathbb{R}$ there exists a unique solution defined on $[0,+\infty[$ to the system (22). Moreover, we have:

- If $d<0$, then for all $t \geq 0$,

$$
\begin{aligned}
& 4|d||A(t)|^{2}+4|S(t)|^{2}+(D(t)-d)^{2} \\
& \leq \mathrm{e}^{-2 t \min \{\kappa, \gamma\}}\left(4|d||A(0)|^{2}+4|S(0)|^{2}+(D(0)-d)^{2}\right) .
\end{aligned}
$$

- If $d \geq 0$, then for any $t \geq 0$,

$$
\begin{aligned}
& |A(t)|^{2}+\frac{g^{2}}{\gamma \kappa}|S(t)|^{2}+\frac{g^{2}}{4 \gamma \kappa}(D(t)-d)^{2} \\
& \leq \mathrm{e}^{-t \min \left\{\kappa-\frac{g^{2} d}{\gamma}, \gamma-\frac{g^{2} d}{\kappa}\right\}}\left(|A(0)|^{2}+\frac{g^{2}}{\gamma \kappa}|S(0)|^{2}+\frac{g^{2}}{4 \gamma \kappa}(D(0)-d)^{2}\right)
\end{aligned}
$$


Proof. Deferred to Subection 5.2 .3 .

Remark 4.2. According to $\gamma=\left(\kappa_{+}+\kappa_{-}\right) / 2, d=\left(\kappa_{+}-\kappa_{-}\right) /\left(\kappa_{+}+\kappa_{-}\right)$we have $\kappa_{-}=\gamma(1-d)$ and $\kappa_{+}=\gamma(1+d)$. Since $\kappa_{+}, \kappa_{-}>0, \gamma>0$ and $\left.d \in\right]-1,1[$.

\section{Proofs}

\subsection{Proofs of theorems from Section 3}

\subsubsection{Proof of Theorem 3.1}

The proof of Theorem 3.1 follows from combining Lemma 5.2, given below, with the arguments used in the proof of Theorem 4.4 of [37]. First, we get the weak continuity of the map $t \mapsto A X_{t}(\xi)$ in case $A$ is relatively bounded by $C$.

Lemma 5.1. Let Condition H2.3 of Hypothesis 2 hold. Suppose that $\xi \in L_{C}^{2}(\mathbb{P}, \mathfrak{h})$ and $A \in \mathfrak{L}\left(\left(\mathcal{D}(C),\|\cdot\|_{C}\right), \mathfrak{h}\right)$. Then, for any $\psi \in L^{2}(\mathbb{P}, \mathfrak{h})$ and $t \geq 0$ we have

$$
\lim _{s \rightarrow t} \mathbb{E}\left\langle\psi, A X_{s}(\xi)\right\rangle=\mathbb{E}\left\langle\psi, A X_{t}(\xi)\right\rangle .
$$

Proof. Consider a sequence of non-negative real numbers $\left(s_{n}\right)_{n}$ satisfying $s_{n} \rightarrow t$ as $n \rightarrow+\infty$. Since $\left(\left(X_{s_{n}}(\xi), A X_{s_{n}}(\xi), C X_{s_{n}}(\xi)\right)\right)_{n}$ is a bounded sequence in $L^{2}\left(\mathbb{P}, \mathfrak{h}^{3}\right)$, where $\mathfrak{h}^{3}=\mathfrak{h} \times \mathfrak{h} \times \mathfrak{h}$, there exists a subsequence $\left(s_{n(k)}\right)_{k}$ such that

$$
\left(X_{s_{n(k)}}(\xi), A X_{s_{n(k)}}(\xi), C X_{s_{n(k)}}(\xi)\right) \longrightarrow_{k \rightarrow \infty}(Y, U, V)
$$

weakly in $L^{2}\left(\mathbb{P}, \mathfrak{h}^{3}\right)$. Define $\mathfrak{M}=\left\{(\eta, A \eta, C \eta): \eta \in L_{C}^{2}(\mathbb{P}, \mathfrak{h})\right\}$. Thus,

$$
\left(X_{s_{n(k)}}(\xi), A X_{s_{n(k)}}(\xi), C X_{s_{n(k)}}(\xi)\right) \in \mathfrak{M} \quad \forall k \in \mathbb{N} .
$$

Since $\mathfrak{M}$ is a linear manifold of $L^{2}\left(\mathbb{P}, \mathfrak{h}^{3}\right)$ closed with respect to the strong topology (see, e.g., proof of Lemma 7.15 of [37]), (26) implies $(Y, U, V) \in \mathfrak{M}$ (see, e.g., Section

III.1.6 of [29]). Using $\mathbb{E}\left(\sup _{s \in[0, t+1]}\left\|X_{s}(\xi)\right\|^{2}\right)<\infty$, together with the dominated convergence theorem we obtain that

$$
\mathbb{E}\left\|X_{s_{n(k)}}(\xi)-X_{t}(\xi)\right\|^{2} \rightarrow 0 \quad \text { as } k \rightarrow+\infty .
$$

Hence $Y=X_{t}(\xi)$, and so $U=A X_{t}(\xi)$. Therefore, $A X_{s_{n(k)}}(\xi)$ converges to $A X_{t}(\xi)$ weakly in $L^{2}(\mathbb{P}, \mathfrak{h})$.

Lemma 5.2. Assume Hypothesis 圆, together with $\xi \in L_{C}^{2}(\mathbb{P}, \mathfrak{h})$ and $A \in \mathfrak{L}(\mathfrak{h})$. Then, $t \mapsto L_{k}(t) X_{t}(\xi)$ is continuous as a map from $\left[0,+\infty\left[\right.\right.$ to $L^{2}(\mathbb{P}, \mathfrak{h})$. Moreover,

$$
\begin{aligned}
t \mapsto \mathbb{E}\left\langle G(t) X_{t}(\xi), A X_{t}(\xi)\right\rangle+\mathbb{E}\left\langle X_{t}(\xi), A G(t) X_{t}(\xi)\right\rangle \\
+\sum_{\ell=1}^{\infty} \mathbb{E}\left\langle L_{\ell}(t) X_{t}(\xi), A L_{\ell}(t) X_{t}(\xi)\right\rangle
\end{aligned}
$$

is a continuous function. 
Proof. Suppose that $\left(t_{n}\right)_{n}$ is a sequence of non-negative real numbers satisfying $t_{n} \rightarrow t$ as $n \rightarrow+\infty$. By $\mathbb{E}\left(\sup _{s \in[0, t+1]}\left\|X_{s}(\xi)\right\|^{2}\right)<\infty$ (see, e.g., Th. 4.2 .5 of [44]), using the dominated convergence theorem gives

$$
\mathbb{E}\left\|X_{t_{n}}(\xi)-X_{t}(\xi)\right\|^{2} \longrightarrow_{n \rightarrow+\infty} 0
$$

and hence $A X_{t_{n}}(\xi) \longrightarrow_{n \rightarrow \infty} A X_{t}(\xi)$ in $L^{2}(\mathbb{P}, \mathfrak{h})$. For any $\psi \in L^{2}(\mathbb{P}, \mathfrak{h})$,

$$
\begin{aligned}
& \left|\mathbb{E}\left\langle\psi, G(s) X_{s}(\xi)\right\rangle-\mathbb{E}\left\langle\psi, G(t) X_{t}(\xi)\right\rangle\right| \\
\leq \quad & \mathbb{E}\|\psi\|\left\|G(s) X_{s}(\xi)-G(t) X_{s}(\xi)\right\| \\
+ & \left|\mathbb{E}\left\langle\psi, G(t) X_{s}(\xi)\right\rangle-\mathbb{E}\left\langle\psi, G(t) X_{t}(\xi)\right\rangle\right|,
\end{aligned}
$$

and so combining Lemma 5.1 with

$$
\mathbb{E}\left\|G(s) X_{s}(\xi)-G(t) X_{s}(\xi)\right\|^{2} \leq f_{0}(s, t) \mathbb{E}\left\|X_{s}(\xi)\right\|_{C}^{2}
$$

yields

$$
\lim _{s \rightarrow t} \mathbb{E}\left\langle\psi, G(s) X_{s}(\xi)\right\rangle=\mathbb{E}\left\langle\psi, G(t) X_{t}(\xi)\right\rangle
$$

Therefore

$$
\lim _{n \rightarrow \infty} \mathbb{E}\left\langle G\left(t_{n}\right) X_{t_{n}}(\xi), A X_{t_{n}}(\xi)\right\rangle=\mathbb{E}\left\langle G(t) X_{t}(\xi), A X_{t}(\xi)\right\rangle
$$

(see, e.g., Section III.1.7 of [29]). Analysis similar to that in (27) shows

$$
\lim _{s \rightarrow t} \mathbb{E}\left\langle\psi, L_{\ell}(s) X_{s}(\xi)\right\rangle=\mathbb{E}\left\langle\psi, L_{\ell}(t) X_{t}(\xi)\right\rangle,
$$

and hence

$$
L_{\ell}\left(t_{n}\right) X_{t_{n}}(\xi) \longrightarrow_{n \rightarrow \infty} L_{\ell}(t) X_{t}(\xi) \quad \text { weakly in } L^{2}(\mathbb{P}, \mathfrak{h}) .
$$

According to (28) with $A$ replaced by $A^{*}$ we have the continuity of the function $t \mapsto \mathbb{E}\left\langle A^{*} X_{t}(\xi), G(t) X_{t}(\xi)\right\rangle$, and so $t \mapsto \mathbb{E}\left\langle X_{t}(\xi), A G(t) X_{t}(\xi)\right\rangle$ is continuous. Moreover, taking $A=I$ in (28) we deduce that

$$
\mathbb{E} \Re\left\langle X_{t_{n}}(\xi), G\left(t_{n}\right) X_{t_{n}}(\xi)\right\rangle \rightarrow_{n \rightarrow \infty} \mathbb{E} \Re\left\langle X_{t}(\xi), G(t) X_{t}(\xi)\right\rangle .
$$

Applying Condition H2.2 we now get

$$
\sum_{\ell=1}^{\infty} \mathbb{E}\left\|L_{\ell}\left(t_{n}\right) X_{t_{n}}(\xi)\right\|^{2} \longrightarrow_{n \rightarrow \infty} \sum_{\ell=1}^{\infty} \mathbb{E}\left\|L_{\ell}(t) X_{t}(\xi)\right\|^{2} .
$$

Combining (29) and (30) yields

$$
\limsup _{n \rightarrow \infty} \mathbb{E}\left\|L_{\ell}\left(t_{n}\right) X_{t_{n}}(\xi)\right\|^{2} \leq \mathbb{E}\left\|L_{\ell}(t) X_{t}(\xi)\right\|^{2}
$$

(see, e.g., proof of Lemma 7.16 of [37] for details) which, together with (29), implies that $L_{\ell}\left(t_{n}\right) X_{t_{n}}(\xi)$ converges strongly in $L^{2}(\mathbb{P}, \mathfrak{h})$ to $L_{\ell}(t) X_{t}(\xi)$ as $n \rightarrow \infty$. Therefore, $t \mapsto L_{\ell}(t) X_{t}(\xi)$ is continuous as a function from $\left[0,+\infty\left[\right.\right.$ to $L^{2}(\mathbb{P}, \mathfrak{h})$. 
Using Condition H2.2 we obtain that $\sum_{\ell=1}^{n} \mathbb{E}\left\langle L_{\ell}(t) X_{t}(\xi), A L_{\ell}(t) X_{t}(\xi)\right\rangle$ converges to $\sum_{\ell=1}^{\infty} \mathbb{E}\left\langle L_{\ell}(t) X_{t}(\xi), A L_{\ell}(t) X_{t}(\xi)\right\rangle$ as $n \rightarrow \infty$ uniformly on any finite interval. Since

$$
\mathbb{E}\left\langle L_{\ell}\left(t_{n}\right) X_{t_{n}}(\xi), A L_{\ell}\left(t_{n}\right) X_{t_{n}}(\xi)\right\rangle \longrightarrow_{n \rightarrow \infty} \mathbb{E}\left\langle L_{\ell}(t) X_{t}(\xi), A L_{\ell}(t) X_{t}(\xi)\right\rangle,
$$

the map $t \mapsto \sum_{\ell=1}^{\infty} \mathbb{E}\left\langle L_{\ell}(t) X_{t}(\xi), A L_{\ell}(t) X_{t}(\xi)\right\rangle$ is continuous.

Lemma 5.3. Let Hypothesis $⿴ 囗 2$ hold, except Condition H2.4. For any $\xi \in L_{C}^{2}(\mathbb{P}, \mathfrak{h})$, we define

$$
\begin{aligned}
\mathcal{L}_{*}(\xi, t)= & \mathbb{E}\left|G(t) X_{t}(\xi)\right\rangle\left\langle X_{t}(\xi)|+\mathbb{E}| X_{t}(\xi)\right\rangle\left\langle G(t) X_{t}(\xi)\right| \\
& +\sum_{\ell=1}^{\infty} \mathbb{E}\left|L_{\ell}(t) X_{t}(\xi)\right\rangle\left\langle L_{\ell}(t) X_{t}(\xi)\right|
\end{aligned}
$$

Then $\mathcal{L}_{*}(\xi, t)$ is a trace-class operator on $\mathfrak{h}$ whose trace-norm is uniformly bounded with respect to $t$ on bounded time intervals; the series involved in the definition of $\mathcal{L}_{*}$ converges in $\mathfrak{L}_{1}(\mathfrak{h})$.

Proof. By Condition H2.2, using $\||x\rangle\langle y|\left\|_{1}=\right\| x\|\| y \|$ and Lemma 7.3 of [37] we get

$$
\begin{gathered}
\| \mathbb{E}\left|G(t) X_{t}(\xi)\right\rangle\left\langle X_{t}(\xi)\left|\left\|_{1}+\right\| \mathbb{E}\right| X_{t}(\xi)\right\rangle\left\langle G(t) X_{t}(\xi)\right| \|_{1} \\
+\sum_{\ell=1}^{\infty} \| \mathbb{E}\left|L_{\ell}(t) X_{t}(\xi)\right\rangle\left\langle L_{\ell}(t) X_{t}(\xi)\right| \|_{1} \\
\leq 4 \mathbb{E}\left(\left\|X_{t}(\xi)\right\|\left\|G(t) X_{t}(\xi)\right\|\right) \leq K(t) \sqrt{\mathbb{E}\|\xi\|^{2}} \sqrt{\mathbb{E}\left\|X_{t}(\xi)\right\|_{C}^{2}},
\end{gathered}
$$

where the last inequality follows from Condition H2.1.

Applying Lemma 7.3 of [37] and Lemma 5.2 we easily obtain Lemma 5.4.

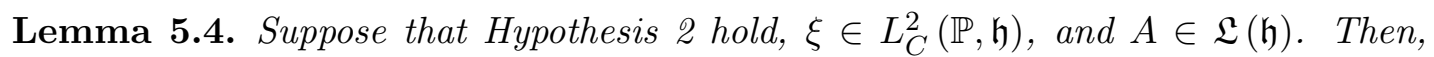
$t \mapsto \operatorname{tr}\left(A \mathcal{L}_{*}(\xi, t)\right)$ is continuous as a function from $[0, \infty[$ to $\mathbb{C}$, and

$$
\begin{aligned}
\operatorname{tr}\left(A \mathcal{L}_{*}(\xi, t)\right)= & \mathbb{E}\left\langle X_{t}(\xi), A G(t) X_{t}(\xi)\right\rangle+\mathbb{E}\left\langle G(t) X_{t}(\xi), A X_{t}(\xi)\right\rangle \\
& +\sum_{\ell=1}^{\infty} \mathbb{E}\left\langle L_{\ell}(t) X_{t}(\xi), A L_{\ell}(t) X_{t}(\xi)\right\rangle .
\end{aligned}
$$

Here, $\mathcal{L}_{*}(\xi, t)$ is as in Lemma 5.3.

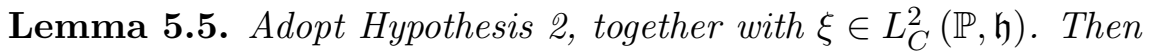

$$
\rho_{t}=\mathbb{E}|\xi\rangle\langle\xi|+\int_{0}^{t} \mathcal{L}_{*}(\xi, s) d s,
$$

where $t \geq 0$ and $\mathcal{L}_{*}(\xi, s)$ is as in Lemma 5.3; we understand the above integral in the sense of Bochner integral in $\mathfrak{L}_{1}(\mathfrak{h})$. 
Proof. Fix $x \in \mathfrak{h}$, and choose $\tau_{n}=\inf \left\{s \geq 0:\left\|X_{s}(\xi)\right\|>n\right\}$, with $n \in \mathbb{N}$. Applying the complex Itô formula we obtain that

$$
\left\langle X_{t \wedge \tau_{n}}(\xi), x\right\rangle X_{t \wedge \tau_{n}}(\xi)=\langle\xi, x\rangle \xi+\mathbb{E} \int_{0}^{t \wedge \tau_{n}} L_{x}\left(X_{s}(\xi), s\right) d s+M_{t},
$$

where

$$
M_{t}=\sum_{\ell=1}^{\infty} \int_{0}^{t \wedge \tau_{n}}\left(\left\langle X_{s}(\xi), x\right\rangle L_{\ell}(s) X_{s}(\xi)+\left\langle L_{\ell}(s) X_{s}(\xi), x\right\rangle X_{s}(\xi)\right) d W_{s}^{\ell}
$$

and for any $z \in \mathcal{D}(C)$,

$$
L_{x}(z, s)=\langle z, x\rangle G(s) z+\langle G(s) z, x\rangle z+\sum_{k=1}^{\infty}\left\langle L_{k}(s) z, x\right\rangle L_{k}(s) z .
$$

According to Condition H2.2 we have

$$
\begin{aligned}
& \mathbb{E} \sum_{\ell=1}^{\infty} \int_{0}^{t \wedge \tau_{n}}\left\|\left\langle X_{s}(\xi), x\right\rangle L_{\ell}(s) X_{s}(\xi)+\left\langle L_{\ell}(s) X_{s}(\xi), x\right\rangle X_{s}(\xi)\right\|^{2} d s \\
& \leq 4 n^{3}\|x\|^{2} \mathbb{E} \int_{0}^{t \wedge \tau_{n}}\left\|G(s) X_{s}\right\| d s .
\end{aligned}
$$

Therefore $\mathbb{E} M_{t}=0$ by Condition H2.1, and so (32) yields

$$
\mathbb{E}\left\langle X_{t \wedge \tau_{n}}(\xi), x\right\rangle X_{t \wedge \tau_{n}}(\xi)=\mathbb{E}\langle\xi, x\rangle \xi+\mathbb{E} \int_{0}^{t \wedge \tau_{n}} L_{x}\left(X_{s}(\xi), s\right) d s .
$$

We will take the limit as $n \rightarrow \infty$ in (33). Since $X(\xi)$ has continuous sample paths, $\tau_{n} \nearrow_{n \rightarrow \infty} \infty$. By H2.1 and H2.2, applying the dominated convergence yields

$$
\lim _{n \rightarrow \infty} \mathbb{E} \int_{0}^{t \wedge \tau_{n}} L_{x}\left(X_{s}(\xi), s\right) d s=\mathbb{E} \int_{0}^{t} L_{x}\left(X_{s}(\xi), s\right) d s .
$$

Combining $\mathbb{E}\left(\sup _{s \in[0, t+1]}\left\|X_{s}(\xi)\right\|^{2}\right)<\infty$ with the dominated convergence theorem gives $\lim _{n \rightarrow \infty} \mathbb{E}\left\langle X_{t \wedge \tau_{n}}(\xi), x\right\rangle X_{t \wedge \tau_{n}}(\xi)=\mathbb{E}\left\langle X_{t}(\xi), x\right\rangle X_{t}(\xi)$. Then, letting first $n \rightarrow$ $\infty$ in (33) and then using Fubini's theorem we get

$$
\mathbb{E}\left\langle X_{t}(\xi), x\right\rangle X_{t}(\xi)=\mathbb{E}\langle\xi, x\rangle \xi+\int_{0}^{t} \mathbb{E} L_{x}\left(X_{s}(\xi), s\right) .
$$

By Condition H2.2, the dominated convergence theorem leads to

$$
\mathbb{E} \sum_{k=1}^{\infty}\left\langle L_{k}(s) X_{s}(\xi), x\right\rangle L_{k}(s) X_{s}(\xi)=\sum_{k=1}^{\infty} \mathbb{E}\left\langle L_{k}(s) X_{s}(\xi), x\right\rangle L_{k}(s) X_{s}(\xi),
$$

and so Lemma 7.3 of [37] yields $\mathbb{E} L_{x}\left(X_{s}(\xi), s\right)=\mathcal{L}_{*}(\xi, s) x$, hence

$$
\int_{0}^{t} \mathbb{E} L_{x}\left(X_{s}(\xi), s\right)=\int_{0}^{t} \mathcal{L}_{*}(\xi, s) x d s .
$$


Since the dual of $\mathfrak{L}_{1}(\mathfrak{h})$ consists in all linear maps $\varrho \mapsto \operatorname{tr}(A \varrho)$ with $A \in \mathfrak{L}(\mathfrak{h})$, Lemma 5.4 implies that $t \mapsto \mathcal{L}_{*}(\xi, t)$ is measurable as a function from $\left[0, \infty\left[\right.\right.$ to $\mathfrak{L}_{1}(\mathfrak{h})$. Furthermore, using Lemma 5.3 we get that $t \mapsto \mathcal{L}_{*}(\xi, t)$ is a Bochner integrable $\mathfrak{L}_{1}(\mathfrak{h})$ valued function on bounded intervals. Then (34), together with (35), gives (31).

Proof. (of Theorem 3.1) According to Theorem 3.2 of [37] we have

$$
A G(t) \rho_{t}=\mathbb{E}\left|A G(t) X_{t}(\xi)\right\rangle\left\langle X_{t}(\xi)\right| .
$$

Since $G(t), L_{1}(t), L_{2}(t), \ldots$ are closable, $G(t)^{*}, L_{1}(t)^{*}, L_{2}(t)^{*}, \ldots$ are densely defined and $G(t)^{* *}, L_{1}(t)^{* *}, \ldots$ coincide with the closures of $G(t), L_{1}(t), \ldots$ respectively (see, e.g., Theorem III.5.29 of [29]). Now, Theorem 3.2 of [37] yields $A \rho_{t} G(t)^{*}=$ $\mathbb{E}\left|A X_{t}(\xi)\right\rangle\left\langle G(t) X_{t}(\xi)\right|$ and

$$
A L_{k}(t) \rho_{t} L_{k}(t)^{*}=\mathbb{E}\left|A L_{k}(t) X_{t}(\xi)\right\rangle\left\langle L_{k}(t) X_{t}(\xi)\right| .
$$

Therefore

$$
\mathcal{L}_{*}(\xi, t)=G(t) \rho_{t}+\rho_{t} G(t)^{*}+\sum_{k=1}^{\infty} L_{k}(t) \rho_{t} L_{k}(t)^{*},
$$

where $\mathcal{L}_{*}(\xi, t)$ is as in Lemma 5.3, Combining (36) with Lemma 5.5 we get (10), and so $\operatorname{tr}\left(A \rho_{t}\right)=\operatorname{tr}(A \varrho)+\int_{0}^{t} \operatorname{tr}\left(A \mathcal{L}_{*}(\xi, s)\right) d s$ for all $t \geq 0$. Using the continuity of $\mathcal{L}_{*}(\xi, \cdot)$ we obtain (9).

\subsubsection{Proof of Theorem 3.2}

Proof. For any $x, y \in \mathcal{D}(C)$ we set $[x, y]=\mathbb{E}\left\langle X_{t}(x), A X_{t}(y)\right\rangle$. According to Definition 3.1 we have

$$
|[x, y]|=\left|\mathbb{E}\left\langle X_{t}(x), A X_{t}(y)\right\rangle\right| \leq K(t)\|A\|\|x\|\|y\| \quad \forall x, y \in \mathcal{D}(C) .
$$

Since $\mathcal{D}(C)$ is dense in $\mathfrak{h},[\cdot, \cdot]$ can be extended uniquely to a sesquilinear form $[\cdot, \cdot]$ over $\mathfrak{h} \times \mathfrak{h}$ satisfying $|[x, y]| \leq K(t)\|A\|\|x\|\|y\|$ for any $x, y \in \mathfrak{h}$. Hence, there exists a unique bounded operator $\mathcal{T}_{t}(A)$ on $\mathfrak{h}$ such that $|[x, y]|=\left\langle x, \mathcal{T}_{t}(A) y\right\rangle$ for all $x, y$ in $\mathfrak{h}$. Moreover, $\left\|\mathcal{T}_{t}(A)\right\| \leq K(t)\|A\|$.

\subsubsection{Proof of Theorem 3.3}

Proof. Using Itô's formula we will prove that for all $x, y \in \mathcal{D}(C)$,

$$
\mathbb{E}\left\langle X_{t}(x), A X_{t}(y)\right\rangle=\left\langle x, \mathcal{A}_{t} y\right\rangle \text {. }
$$

This, together with Theorem 3.2 , implies $\mathcal{A}_{t}=\mathcal{T}_{t}(A)$.

Motivated by $\mathcal{A}_{t}$ is only a weak solution, we fix an orthonormal basis $\left(e_{n}\right)_{n \in \mathbb{N}}$ of $\mathfrak{h}$ and consider the function $F_{n}:[0, t] \times \mathfrak{h} \times \mathfrak{h} \rightarrow \mathbb{C}$ defined by

$$
F_{n}(s, u, v)=\left\langle R_{n} \bar{u}, \mathcal{A}_{t-s} R_{n} v\right\rangle
$$


where $R_{n}=n(n+C)^{-1}$ and $\bar{u}=\sum_{n \in \mathbb{N}} \overline{\left\langle e_{n}, u\right\rangle} e_{n}$. Since the range of $R_{n}$ is contained in $\mathcal{D}(C)$,

$$
\frac{d}{d s} F_{n}(s, u, v)=-g\left(s, R_{n} \bar{u}, R_{n} v\right),
$$

with $g(s, x, y)=\left\langle x, \mathcal{A}_{t-s} G y\right\rangle+\left\langle G x, \mathcal{A}_{t-s} y\right\rangle+\sum_{k=1}^{\infty}\left\langle L_{k} x, \mathcal{A}_{t-s} L_{k} y\right\rangle$. We have that $t \longmapsto\left\langle u, \mathcal{A}_{t} v\right\rangle$ is continuous for all $u, v \in \mathfrak{h}$, and so combining $C R_{n} \in \mathfrak{L}(\mathfrak{h})$ with Hypothesis 3 we get the uniformly continuity of $(s, u, v) \longmapsto g\left(s, R_{n} \bar{u}, R_{n} v\right)$ on bounded subsets of $[0, t] \times \mathfrak{h} \times \mathfrak{h}$. Then, we can apply Itô's formula to $F_{n}\left(s \wedge \tau_{j}, \overline{X_{s}^{\tau_{j}}(x)}, X_{s}^{\tau_{j}}(y)\right)$, with

$$
\tau_{j}=\inf \left\{t \geq 0:\left\|X_{t}(x)\right\|+\left\|X_{t}(y)\right\|>j\right\} .
$$

Fix $x, y \in \mathcal{D}(C)$. Combining Itô's formula with (38) we deduce that

$$
F_{n}\left(t \wedge \tau_{j}, \overline{X_{t}^{\tau_{j}}(x)}, X_{t}^{\tau_{j}}(y)\right)=F_{n}\left(0, \overline{X_{0}(x)}, X_{0}(y)\right)+I_{t \wedge \tau_{j}}^{n}+M_{t},
$$

where for $s \in[0, t]$ :

$$
\begin{aligned}
M_{s}= & \sum_{k=1}^{\infty} \int_{0}^{s \wedge \tau_{j}}\left\langle R_{n} X_{r}^{\tau_{j}}(x), \mathcal{A}_{t-r} R_{n} L_{k} X_{r}^{\tau_{j}}(y)\right\rangle d W_{r}^{k} \\
& +\sum_{k=1}^{\infty} \int_{0}^{s \wedge \tau_{j}}\left\langle R_{n} L_{k} X_{r}^{\tau_{j}}(x), \mathcal{A}_{t-r} R_{n} X_{r}^{\tau_{j}}(y)\right\rangle d W_{r}^{k}
\end{aligned}
$$

and $I_{s}^{n}=\int_{0}^{s}\left(-g\left(r, R_{n} X_{r}(x), R_{n} X_{r}(y)\right)+g_{n}\left(r, X_{r}(x), X_{r}(y)\right)\right) d r$ with

$$
g_{n}(r, u, v)=\left\langle R_{n} u, \mathcal{A}_{t-r} R_{n} G v\right\rangle+\left\langle R_{n} G u, \mathcal{A}_{t-r} R_{n} v\right\rangle+\sum_{k=1}^{\infty}\left\langle R_{n} L_{k} u, \mathcal{A}_{t-r} R_{n} L_{k} v\right\rangle .
$$

We next establish the martingale property of $M_{s}$. For all $r \in[0, t]$ we have

$$
\left\|R_{n} X_{r}^{\tau_{j}}(x)\right\|^{2}\left\|\mathcal{A}_{t-r}\right\|^{2}\left\|R_{n} L_{k} X_{r}^{\tau_{j}}(y)\right\|^{2} \leq j^{2} \sup _{s \in[0, t]}\left\|\mathcal{A}_{s}\right\|^{2}\left\|L_{k} X_{r}^{\tau_{j}}(y)\right\|^{2} .
$$

By H2.1 and H3.1, $\mathbb{E} \int_{0}^{t \wedge \tau_{j}} \sum_{k=1}^{\infty}\left|\left\langle R_{n} X_{r}^{\tau_{j}}(x), \mathcal{A}_{t-r} R_{n} L_{k} X_{r}^{\tau_{j}}(y)\right\rangle\right|^{2} d s<\infty$. Thus

$$
\left(\sum_{k=1}^{\infty} \int_{0}^{s \wedge \tau_{j}}\left\langle R_{n} X_{r}^{\tau_{j}}(x), \mathcal{A}_{t-r} R_{n} L_{k} X_{r}^{\tau_{j}}(y)\right\rangle d W_{r}^{k}\right)_{s \in[0, t]}
$$

is a martingale. The same conclusion can be draw for

$$
\sum_{k=1}^{\infty} \int_{0}^{s \wedge \tau_{j}}\left\langle R_{n} L_{k} X_{r}^{\tau_{j}}(x), \mathcal{A}_{t-r} R_{n} X_{r}^{\tau_{j}}(y)\right\rangle d W_{r}^{k}
$$

and so $\left(M_{s}\right)_{s \in[0, t]}$ is a martingale. Hence

$$
\mathbb{E}\left\langle R_{n} X_{t}^{\tau_{j}}(x), \mathcal{A}_{t-t \wedge \tau_{j}} R_{n} X_{t}^{\tau_{j}}(y)\right\rangle=\left\langle R_{n} x, \mathcal{A}_{t} R_{n} y\right\rangle+\mathbb{E} I_{t \wedge \tau_{j}}^{n}
$$


We will take the limit as $j \rightarrow \infty$ in (39). Since $\mathbb{E}\left(\sup _{s \in[0, t]}\left\|X_{s}(\xi)\right\|^{2}\right)<\infty$ for $\xi=x, y$ (see, e.g., Th. 4.2.5 of [44]), using the dominated convergence theorem, together with the continuity of $t \longmapsto\left\langle u, \mathcal{A}_{t} v\right\rangle$, we get

$$
\mathbb{E}\left\langle R_{n} X_{t}^{\tau_{j}}(x), \mathcal{A}_{t-t \wedge \tau_{j}} R_{n} X_{t}^{\tau_{j}}(y)\right\rangle \rightarrow_{j \rightarrow \infty} \mathbb{E}\left\langle R_{n} X_{t}(x), A R_{n} X_{t}(y)\right\rangle .
$$

Applying again the dominated convergence theorem yields $\mathbb{E} I_{t \wedge \tau_{j}}^{n} \longrightarrow_{j \rightarrow \infty} \mathbb{E} I_{t}^{n}$, and hence letting $j \rightarrow \infty$ in (39) we deduce that

$$
\begin{aligned}
& \mathbb{E}\left\langle R_{n} X_{t}(x), A R_{n} X_{t}(y)\right\rangle-\left\langle R_{n} x, \mathcal{A}_{t} R_{n} y\right\rangle \\
& =\mathbb{E} \int_{0}^{t}\left(-g\left(s, R_{n} X_{s}(x), R_{n} X_{s}(y)\right)+g_{n}\left(s, X_{s}(x), X_{s}(y)\right)\right) d s .
\end{aligned}
$$

Finally, we take the limit as $n \rightarrow \infty$ in (40). Since $\left\|R_{n}\right\| \leq 1$ and $R_{n}$ tends pointwise to $I$ as $n \rightarrow \infty$, the dominated convergence theorem yields

$$
\lim _{n \rightarrow \infty} \mathbb{E} \int_{0}^{t} g_{n}\left(s, X_{s}(x), X_{s}(y)\right) d s=\mathbb{E} \int_{0}^{t} g\left(s, X_{s}(x), X_{s}(y)\right) d s .
$$

For any $x \in \mathcal{D}(C), \lim _{n \rightarrow \infty} C R_{n} x=C x$. By $\left\|C R_{n} x\right\| \leq\|C x\|$, using the dominated convergence theorem gives

$$
\lim _{n \rightarrow \infty} \mathbb{E} \int_{0}^{t} g\left(s, R_{n} X_{s}(x), R_{n} X_{s}(y)\right) d s=\mathbb{E} \int_{0}^{t} g\left(s, X_{s}(x), X_{s}(y)\right) d s .
$$

Thus, letting $n \rightarrow \infty$ in (40) we obtain (37).

\subsection{Proofs of theorems from Section 4}

\subsubsection{Proof of Theorem 4.1}

Proof. First, we show that $\rho_{t}$ given by (18) is a $N^{p}$-weak solution to (14). To this end, we will verify that $C=N^{p}$ satisfies Hypothesis 2, where, here and subsequently, $H(t), G(t), L_{1}, L_{2}, L_{3}$ are defined as in Theorem 4.1. Since $L_{2}, L_{3} \in \mathfrak{L}\left(\ell^{2}\left(\mathbb{Z}_{+}\right) \otimes \mathbb{C}^{2}\right)$, $L_{1}, L_{1}^{*} L_{1}$ are relatively bounded with respect to $N$ and

$$
\|H(t) x\|^{2} \leq K \max (|\alpha(t)|,|\beta(t)|)\|x\|_{N} \quad \forall x \in \mathcal{D}(N),
$$

$C$ fulfills Condition H2.1 of Hypothesis 2, By definition of $G(t)$ and $L_{\ell}$,

$$
2 \Re\langle x, G(t) x\rangle+\sum_{\ell=1}^{3}\left\|L_{\ell} x\right\|^{2}=0 \quad \forall x \in \mathcal{D}(N),
$$

and hence Condition H2.2 holds. Condition H2.4 follows from the continuity of $\alpha$ and $\beta$.

In order to check Condition H2.3, we denote by $\mathfrak{D}$ the set of all $x \in \ell^{2}\left(\mathbb{Z}_{+}\right) \otimes \mathbb{C}^{2}$ such that $x(n, \eta):=\left\langle e_{n} \otimes e_{\eta}, x\right\rangle$ is equal to 0 for all combinations of $n \in \mathbb{Z}_{+}$and $\eta= \pm$ 
except a finite number. Consider $x \in \mathfrak{D}$. A careful computation yields

$$
\begin{aligned}
& 2 \Re\left\langle N^{2 p} x, G(t) x\right\rangle+\sum_{\ell=1}^{3}\left\|N^{p} L_{\ell} x\right\|^{2} \\
& =\sum_{k \in \mathbb{Z}_{+}, \eta= \pm} 2 \Re(\alpha(t) x(k, \eta) \overline{x(k+1, \eta)}) \sqrt{k+1}\left((k+1)^{2 p}-k^{2 p}\right) \\
& \quad+\sum_{k \in \mathbb{Z}_{+}, \eta= \pm} 2 \kappa|x(k, \eta)|^{2} k\left((k-1)^{2 p}-k^{2 p}\right) .
\end{aligned}
$$

Since

$$
\begin{aligned}
& \sum_{k \in \mathbb{Z}_{+}, \eta= \pm} 2 \Re(\alpha(t) x(k, \eta) \overline{x(k+1, \eta)}) \sqrt{k+1}\left((k+1)^{2 p}-k^{2 p}\right) \\
& \leq 2|\alpha(t)| \sum_{k \in \mathbb{Z}_{+}, \eta= \pm}|x(k, \eta)||x(k+1, \eta)| \phi(k) \\
& \leq 2|\alpha(t)| \sum_{k \in \mathbb{Z}_{+}, \eta= \pm}|x(k, \eta)|^{2} \phi(k)
\end{aligned}
$$

with $\phi(k)=\sqrt{k+1}\left((k+1)^{2 p}-k^{2 p}\right)=\sqrt{k+1} \sum_{j=0}^{2 p-1}\left(\begin{array}{c}2 p \\ j\end{array}\right) k^{j}$,

$$
\begin{aligned}
& \sum_{k \in \mathbb{Z}_{+}, \eta= \pm} 2 \Re(\alpha(t) x(k, \eta) \overline{x(k+1, \eta)}) \sqrt{k+1}\left((k+1)^{2 p}-k^{2 p}\right) \\
\leq & |\alpha(t)| K \sum_{k \in \mathbb{Z}_{+}, \eta= \pm}|x(k, \eta)|^{2}\left(1+k^{2 p-1 / 2}\right) .
\end{aligned}
$$

Combining (41) with (42) we get

$$
2 \Re\left\langle N^{2 p} x, G(t) x\right\rangle+\sum_{\ell=1}^{3}\left\|N^{p} L_{\ell} x\right\|^{2} \leq K|\alpha(t)|\|x\|_{N^{p}}^{2},
$$

and so Condition H4.3 of Hypothesis 4 holds because $\mathfrak{D}$ is a core of $N^{p}$. Then, applying Theorem 2.4 of [19] (see also Theorem 3.4) we obtain that for any initial condition $\xi \in L_{N^{p}}^{2}\left(\mathbb{P}, \ell^{2}\left(\mathbb{Z}_{+}\right) \otimes \mathbb{C}^{2}\right)$ there exists a unique strong $N^{p_{\text {-solution }}}$ of (16), together with

$$
\mathbb{E}\left\|X_{t}(\xi)\right\|_{N^{p}}^{2} \leq K(t) \mathbb{E}\|\xi\|_{N^{p}}^{2} .
$$

Therefore, Condition H2.3 holds, and so we have checked Hypothesis 2 with $C=N^{p}$.

Applying Theorem 3.1 of [37] yields $\varrho=\mathbb{E}|\xi\rangle\langle\xi|$ for certain

$$
\xi \in L_{N^{p}}^{2}\left(\mathbb{P}, \ell^{2}\left(\mathbb{Z}_{+}\right) \otimes \mathbb{C}^{2}\right) .
$$

Using Theorem 3.1 we obtain that $\rho_{t}:=\mathbb{E}\left|X_{t}(\xi)\right\rangle\left\langle X_{t}(\xi)\right|$ satisfies the relation (17) and

$$
\begin{cases}\frac{d}{d t} \operatorname{tr}\left(A \rho_{t}\right) & =\operatorname{tr}\left(A\left(G(t) \rho_{t}+\rho_{t} G(t)^{*}+\sum_{\ell=1}^{3} L_{\ell} \rho_{t} L_{\ell}^{*}\right)\right) \\ \rho_{0} & =\varrho\end{cases}
$$


for all $A \in \mathfrak{L}\left(\ell^{2}\left(\mathbb{Z}_{+}\right) \otimes \mathbb{C}^{2}\right)$.

Second, we will prove that (14) has at most one $N^{p}$-weak solution provided that the initial condition is $N^{p}$-regular. Suppose that (44) holds. Taking $A=|y\rangle\langle x|$ in (44) we get

$$
\frac{d}{d t}\left\langle x, \rho_{t} y\right\rangle=\left\langle G(t)^{*} x, \rho_{t} y\right\rangle+\left\langle x, \rho_{t} G(t)^{*} y\right\rangle+\sum_{\ell=1}^{3}\left\langle L_{\ell}^{*} x, \rho_{t} L_{\ell}^{*} y\right\rangle
$$

for all $x, y \in \mathcal{D}\left(N^{p}\right)$. Relation (45) coincides with (13) with $\mathcal{A}_{t}, G(t), L_{1}, L_{2}$ and $L_{3}$ replaced by $\rho_{t}, G(t)^{*}, L_{1}^{*}, L_{2}^{*}$ and $L_{3}^{*}$. This suggests us to apply Theorem 3.3 to (45) in order to prove the uniqueness of the solution of (44). To this end, we next deduce that the linear stochastic Schrödinger equation

$$
Y_{t}(\xi)=\xi+\int_{0}^{t} G(s)^{*} Y_{s}(\xi) d s+\sum_{\ell=1}^{3} \int_{0}^{t} L_{\ell}^{*} Y_{s}(\xi) d W_{s}^{\ell}
$$

satisfies Hypothesis 4 with $C=N^{p}$.

Now, we check Hypothesis 4 with $G(t), L_{1}, L_{2}$ and $L_{3}$ replaced by $G(t)^{*}, L_{1}^{*}, L_{2}^{*}$ and $L_{3}^{*}$. Take $C=N^{p}$. Since $a^{\dagger}$ is relatively bounded with respect to $N$, using analysis similar to that in the second paragraph we can check that $G(t)^{*}=\mathrm{i} H(t)-\frac{1}{2} \sum_{\ell=1}^{3} L_{\ell}^{*} L_{\ell}$ satisfies Condition H4.1 of Hypothesis 4 with $G(t)$ substituted by $G(t)^{*}$, as well as Condition H4.2 holds with $L_{\ell}(t)$ replaced by $L_{1}^{*}=\sqrt{2 \kappa} a^{\dagger}, L_{2}^{*}=\sqrt{\gamma(1-d)} \sigma^{+}, L_{3}^{*}=$ $\sqrt{\gamma(1+d)} \sigma^{-}$. On $\mathfrak{D}$ we have

$$
\begin{aligned}
G(t)^{*}+\left(G(t)^{*}\right)^{*}+\sum_{\ell=1}^{3}\left(L_{\ell}^{*}\right)^{*} L_{\ell}^{*} & =\sum_{\ell=1}^{3}\left(L_{\ell} L_{\ell}^{*}-L_{\ell}^{*} L_{\ell}\right) \\
& =4 \kappa^{2} I+2 \gamma^{2}\left(1+d^{2}\right) \sigma_{3},
\end{aligned}
$$

which gives Condition H4.4. For any $x \in \mathfrak{D}$,

$$
\begin{aligned}
& 2 \Re\left\langle N^{2 p} x, \mathrm{i} H(t) x\right\rangle \\
& =\sum_{k \in \mathbb{Z}_{+}, \eta= \pm} 2 \Re(\alpha(t) x(k, \eta) \overline{x(k+1, \eta)}) \sqrt{k+1}\left((k+1)^{2 p}-k^{2 p}\right)
\end{aligned}
$$

and

$$
\begin{aligned}
& \left\langle x,\left(L_{1} N^{2 p} L_{1}^{*}-\frac{1}{2} L_{1}^{*} L_{1} N^{2 p}-\frac{1}{2} N^{2 p} L_{1}^{*} L_{1}\right) x\right\rangle \\
& =\sum_{k \in \mathbb{Z}_{+}, \eta= \pm} 2 \kappa|x(k, \eta)|^{2}\left((k+1)^{2 p+1}-k^{2 p+1}\right) .
\end{aligned}
$$

Since $L_{2}, L_{3}$ are bounded operators with conmute with $N^{2 p}$, using (47) and (48) yields

$$
2 \Re\left\langle N^{2 p} x, G(t)^{*} x\right\rangle+\sum_{\ell=1}^{3}\left\|N^{p} L_{\ell}^{*} x\right\|^{2} \leq K(t)\left\|N^{p} x\right\|^{2}
$$

and hence Condition H4.3 holds. By Theorem [3.4, (46) has a unique strong $N^{p_{-}}$ solution whenever $\xi \in L_{C}^{2}\left(\mathbb{P}, \ell^{2}\left(\mathbb{Z}_{+}\right) \otimes \mathbb{C}^{2}\right)$. It follows from Theorem 3.3 that (45) has at most one solution $\varrho_{t} \in \mathfrak{L}\left(\ell^{2}\left(\mathbb{Z}_{+}\right) \otimes \mathbb{C}^{2}\right)$ satisfying $\varrho_{0}=\varrho$. Thus, (14) has a unique $N^{p}$-regular solution, which is equal to $\rho_{t}:=\mathbb{E}\left|X_{t}(\xi)\right\rangle\left\langle X_{t}(\xi)\right|$. 


\subsubsection{Proof of Theorem 4.2}

Proof. From Theorem 4.1 it follows that (16) has a unique strong $N^{p}$-solution $X_{t}(\xi)$ for any initial datum $\xi \in L_{N^{p}}^{2}\left(\mathbb{P}, \ell^{2}\left(\mathbb{Z}_{+}\right) \otimes \mathbb{C}^{2}\right)$. In order to establish (19) we apply Theorem 4.1 of [19] to obtain

$$
\begin{aligned}
\operatorname{tr}\left(a \rho_{t}\right)= & \operatorname{tr}\left(a \rho_{0}\right)+\sum_{\ell=1}^{3} \int_{0}^{t} \mathbb{E}\left\langle L_{\ell} X_{s}(\xi), a L_{\ell} X_{s}(\xi)\right\rangle d s \\
& +\int_{0}^{t}\left(\mathbb{E}\left\langle a^{\dagger} X_{s}(\xi), G(s) X_{s}(\xi)\right\rangle+\mathbb{E}\left\langle G(s) X_{s}(\xi), a X_{s}(\xi)\right\rangle\right) d s,
\end{aligned}
$$

where, throughout the proof, $G(t), H(t), L_{1}, L_{2}, L_{3}$ are as in Theorem 4.1. Therefore, $t \mapsto \operatorname{tr}\left(a \rho_{t}\right)$ is a continuous function.

Suppose that $x \in \mathfrak{D}$, where $\mathfrak{D}$ is the set of all $x \in \ell^{2}\left(\mathbb{Z}_{+}\right) \otimes \mathbb{C}^{2}$ satisfying $\left\langle e_{n} \otimes e_{\eta}, x\right\rangle=$ 0 for all combinations of $n \in \mathbb{Z}_{+}$and $\eta= \pm$ except a finite number. Since $a$ conmutes with $\sigma^{3}$ and $\sigma^{ \pm}$, using $\left[a, a^{\dagger}\right]=I$ we deduce that

$$
\begin{aligned}
\left\langle a^{\dagger} x,-\mathrm{i} H(s) x\right\rangle+\langle-\mathrm{i} H(s) x, a x\rangle & =\langle x, \mathrm{i}[H(s), a] x\rangle \\
& =\left\langle x,\left[\mathrm{i} \omega a^{\dagger} a-\alpha(t) a^{\dagger}+\overline{\alpha(t)} a, a\right] x\right\rangle \\
& =\langle x,(-\mathrm{i} \omega a+\alpha(t)) x\rangle
\end{aligned}
$$

and

$$
\begin{aligned}
& \sum_{\ell=1}^{3}\left\langle x,\left(L_{\ell}^{\star} a L_{\ell}-\frac{1}{2} a L_{\ell}^{\star} L_{\ell}-\frac{1}{2} L_{\ell}^{\star} L_{\ell} a\right) x\right\rangle \\
& =\left\langle x,\left(L_{1}^{\star} a L_{1}-\frac{1}{2} a L_{1}^{\star} L_{1}-\frac{1}{2} L_{1}^{\star} L_{1} a\right) x\right\rangle=-\kappa\langle x, a x\rangle .
\end{aligned}
$$

Because $\mathfrak{D}$ is a core for $N$, we obtain that for all $x \in \mathcal{D}(N)$,

$$
\left\langle a^{\dagger} x, G(s) x\right\rangle+\langle G(s) x, a x\rangle+\sum_{\ell=1}^{3}\left\langle L_{\ell} x, a L_{\ell} x\right\rangle=\langle x,-(\kappa+\mathrm{i} \omega) a x+\alpha(t) x\rangle .
$$

Then, from (49) it follows that

$$
\operatorname{tr}\left(a \rho_{t}\right)=\operatorname{tr}\left(a \rho_{0}\right)+\int_{0}^{t}\left(-(\kappa+\mathrm{i} \omega) \operatorname{tr}\left(a \rho_{s}\right)+\alpha(s)\right) d s
$$

which leads to (19).

Fix $\eta=-$ or $\eta=3$. According to (44) we have

$$
\frac{d}{d t} \operatorname{tr}\left(\rho_{t} \sigma^{\eta}\right)=\operatorname{tr}\left(\sigma^{\eta}\left(G(t) \rho_{t}+\rho_{t} G(t)^{*}+\sum_{\ell=1}^{3} L_{\ell} \rho_{t} L_{\ell}^{*}\right)\right),
$$


and so applying Theorem 3.2 of [37] we deduce that

$$
\begin{aligned}
& \frac{d}{d t} \operatorname{tr}\left(\rho_{t} \sigma^{\eta}\right)=\operatorname{tr}\left(\rho_{t}\left(\sigma^{\eta} G(t)+G(t)^{*} \sigma^{\eta}+\sum_{\ell=1}^{3} L_{\ell}^{*} \sigma^{\eta} L_{\ell}\right)\right) \\
& =\operatorname{tr}\left(\rho_{t}\left(-\mathrm{i}\left[\sigma^{\eta}, H(t)\right]+\sum_{\ell=1}^{3}\left(L_{\ell}^{*} \sigma^{\eta} L_{\ell}-\frac{1}{2} \sigma^{\eta} L_{\ell}^{*} L_{\ell}-\frac{1}{2} L_{\ell}^{*} L_{\ell} \sigma^{\eta}\right)\right)\right) \\
& =\operatorname{tr}\left(-\mathrm{i} \rho_{t}\left[\sigma^{\eta}, \frac{\omega}{2} \sigma^{3}+\mathrm{i}\left(\overline{\beta(t)} \sigma^{-}-\beta(t) \sigma^{+}\right)\right]\right) \\
& \quad+\sum_{\ell=2}^{3} \operatorname{tr}\left(\rho_{t}\left(\left(L_{\ell}^{*} \sigma^{\eta} L_{\ell}-\frac{1}{2} \sigma^{\eta} L_{\ell}^{*} L_{\ell}-\frac{1}{2} L_{\ell}^{*} L_{\ell} \sigma^{\eta}\right)\right)\right) .
\end{aligned}
$$

Now, we use the commutation relations

$$
\left[\sigma^{+}, \sigma^{-}\right]=\sigma^{3}, \quad\left[\sigma^{3}, \sigma^{+}\right]=2 \sigma^{+}, \quad\left[\sigma^{-}, \sigma^{3}\right]=2 \sigma^{-}
$$

to derive (20) and (21).

\subsubsection{Proof of Theorem 4.3}

Proof. Fix $A(0) \in \mathbb{C}, S(0) \in \mathbb{C}$ and $D(0) \in \mathbb{R}$. Since (22) is an ordinary differential equation with locally Lipschitz coefficients, (22) has a unique solution defined on a maximal interval $[0, T[$ (see, e.g., [28]).

For all $t \in[0, T$, we set $X(t)=\exp (i \omega t) A(t), Y(t)=\exp (i \omega t) S(t)$ and $Z(t)=$ $D(t)-d$. Thus, (22) becomes

$$
\left\{\begin{array}{l}
X^{\prime}(t)=-\kappa X(t)+g Y(t) \\
Y^{\prime}(t)=d g X(t)-\gamma Y(t)+g X(t) Z(t) \\
Z^{\prime}(t)=-4 g \Re(\overline{X(t)} Y(t))-2 \gamma Z(t)
\end{array}\right.
$$

Therefore,

$$
\frac{d}{d t}|X(t)|^{2}=2 \Re\left(X^{\prime}(t) \overline{X(t)}\right)=-2 \kappa|X(t)|^{2}+2 g \Re(Y(t) \overline{X(t)})
$$

and

$$
\left\{\begin{array}{ll}
\frac{d}{d t}|Y(t)|^{2} & =2 d g \Re(X(t) \overline{Y(t)})-2 \gamma|Y(t)|^{2}+2 g Z(t) \Re(X(t) \overline{Y(t)}) \\
\frac{d}{d t} Z(t)^{2} & =-4 \gamma Z(t)^{2}-8 g Z(t) \Re(\overline{X(t)} Y(t))
\end{array} .\right.
$$

Hence,

$$
4 \frac{d}{d t}|Y(t)|^{2}+\frac{d}{d t} Z(t)^{2}=8 d g \Re(X(t) \overline{Y(t)})-8 \gamma|Y(t)|^{2}-4 \gamma Z(t)^{2} .
$$

Suppose, for a moment, that $d<0$. Then

$$
-4 d \frac{d}{d t}|X(t)|^{2}+4 \frac{d}{d t}|Y(t)|^{2}+\frac{d}{d t} Z(t)^{2}=8 d \kappa|X(t)|^{2}-8 \gamma|Y(t)|^{2}-4 \gamma Z(t)^{2} .
$$


This gives

$$
\begin{aligned}
& \frac{d}{d t}\left(-4 d|X(t)|^{2}+4|Y(t)|^{2}+(Z(t))^{2}\right) \\
& \leq-\min \{2 \kappa, 2 \gamma\}\left(-4 d|X(t)|^{2}+4|Y(t)|^{2}+Z(t)^{2}\right),
\end{aligned}
$$

which implies

$$
\begin{aligned}
& 4|d||X(t)|^{2}+4|Y(t)|^{2}+Z(t)^{2} \\
& \leq \exp (-2 t \min \{\kappa, \gamma\})\left(4|d||X(0)|^{2}+4|Y(0)|^{2}+Z(0)^{2}\right)
\end{aligned}
$$

for any $t \in[0, T[$.

On the other hand, assume that $d \geq 0$. Combining

$$
\begin{aligned}
& \frac{d}{d t}|X(t)|^{2}+\frac{g^{2}}{4 \gamma \kappa}\left(4 \frac{d}{d t}|Y(t)|^{2}+\frac{d}{d t} Z(t)^{2}\right) \\
& =2 g\left(1+\frac{g^{2} d}{\gamma \kappa}\right) \Re(X(t) \overline{Y(t)})-2 \kappa|X(t)|^{2}-2 \frac{g^{2}}{\kappa}|Y(t)|^{2}-\frac{g^{2}}{\kappa} Z(t)^{2}
\end{aligned}
$$

with $2 \Re\left(X(t) \frac{\bar{g}}{\kappa} Y(t)\right) \leq|X(t)|^{2}+\frac{g^{2}}{\kappa^{2}}|Y(t)|^{2}$ we obtain

$$
\begin{aligned}
& \frac{d}{d t}\left(|X(t)|^{2}+\frac{g^{2}}{\gamma \kappa}|Y(t)|^{2}+\frac{g^{2}}{4 \gamma \kappa} Z(t)^{2}\right) \\
& \leq\left(-\kappa+\frac{g^{2} d}{\gamma}\right)|X(t)|^{2}+\left(-\gamma+\frac{g^{2} d}{\kappa}\right) \frac{g^{2}}{\gamma \kappa}|Y(t)|^{2}-4 \gamma \frac{g^{2}}{4 \gamma \kappa} Z(t)^{2} .
\end{aligned}
$$

Therefore, for all $t \in[0, T[$ we have

$$
\begin{aligned}
& \frac{d}{d t}\left(|X(t)|^{2}+\frac{g^{2}}{\gamma \kappa}|Y(t)|^{2}+\frac{g^{2}}{4 \gamma \kappa} Z(t)^{2}\right) \\
& \leq-\min \left\{\kappa-\frac{g^{2} d}{\gamma}, \gamma-\frac{g^{2} d}{\kappa}\right\}\left(|X(t)|^{2}+\frac{g^{2}}{\gamma \kappa}|Y(t)|^{2}+\frac{g^{2}}{4 \gamma \kappa} Z(t)^{2}\right) .
\end{aligned}
$$

This yields

$$
\begin{aligned}
& |X(t)|^{2}+\frac{g^{2}}{\gamma \kappa}|Y(t)|^{2}+\frac{g^{2}}{4 \gamma \kappa} Z(t)^{2} \\
& \leq \mathrm{e}^{-t \min \left\{\kappa-\frac{g^{2} d}{\gamma}, \gamma-\frac{g^{2} d}{\kappa}\right\}}\left(|X(0)|^{2}+\frac{g^{2}}{\gamma \kappa}|Y(0)|^{2}+\frac{g^{2}}{4 \gamma \kappa} Z(0)^{2}\right) .
\end{aligned}
$$

Suppose that $T<+\infty$. According to (51) and (52) we have that

$$
\|(A(t), S(t), D(t))\|<K,
$$

where $K>0$ and $t \in[0, T[$. This contradicts the property

$$
\lim _{t \rightarrow T}\|(A(t), S(t), D(t))\|=\infty .
$$

Therefore, $T=+\infty$. Moreover, (151) and (52) lead to (23) and (24), respectively. 


\subsection{Proof of Theorem 2.1}

Proof. Let $(A(t), S(t), D(t))$ be the unique global solution of (22) with $A(0)=$ $\operatorname{tr}(a \varrho), S(0)=\operatorname{tr}\left(\sigma^{-} \varrho\right)$ and $D(0)=\operatorname{tr}\left(\sigma^{3} \varrho\right)$. According to Theorem 4.1 we have that there exists a unique $N^{p}$-weak solution $\left(\rho_{t}\right)_{t \geq 0}$ to (14) with $\alpha(t)=g S(t)$, $\beta(t)=g A(t)$ and initial datum $\rho_{0}=\varrho$. Moreover, Theorem 4.1 ensures that $\rho_{t}=$ $\mathbb{E}\left|Z_{t}(\xi)\right\rangle\left\langle Z_{t}(\xi)\right|$, where $Z_{t}(\xi)$ is the strong $N^{p}$-solution of (16) with $\alpha(t)=g S(t)$, $\beta(t)=g A(t)$ and initial condition $\xi \in L_{N^{p}}^{2}\left(\mathbb{P}, \ell^{2}\left(\mathbb{Z}_{+}\right) \otimes \mathbb{C}^{2}\right)$ such that $\varrho=\mathbb{E}|\xi\rangle\langle\xi|$. Applying Theorem 4.2 we deduce that the evolutions of $\operatorname{tr}\left(a \rho_{t}\right), \operatorname{tr}\left(\sigma^{-} \rho_{t}\right)$ and $\operatorname{tr}\left(\sigma^{3} \rho_{t}\right)$ are governed by

$$
\left\{\begin{array}{l}
\frac{d}{d t} \operatorname{tr}\left(a \rho_{t}\right)=-(\kappa+\mathrm{i} \omega) \operatorname{tr}\left(a \rho_{t}\right)+g S(t) \\
\frac{d}{d t} \operatorname{tr}\left(\sigma^{-} \rho_{t}\right)=-(\gamma+\mathrm{i} \omega) \operatorname{tr}\left(\sigma^{-} \rho_{t}\right)+g A(t) \operatorname{tr}\left(\sigma^{3} \rho_{t}\right) \\
\frac{d}{d t} \operatorname{tr}\left(\sigma^{3} \rho_{t}\right)=-4 g \Re\left(\overline{A(t)} \operatorname{tr}\left(\sigma^{-} \rho_{t}\right)\right)-2 \gamma\left(\operatorname{tr}\left(\sigma^{3} \rho_{t}\right)-d\right)
\end{array}\right.
$$

From the uniqueness of solution to (53) we find $\operatorname{tr}\left(a \rho_{t}\right)=A(t), \operatorname{tr}\left(\sigma^{-} \rho_{t}\right)=S(t)$ and $\operatorname{tr}\left(\sigma^{3} \rho_{t}\right)=D(t)$. Hence

$$
\begin{cases}\frac{d}{d t} \operatorname{tr}\left(A \rho_{t}\right) & =\operatorname{tr}\left(A \mathcal{L}_{\star}\left(\rho_{t}\right) \rho_{t}\right) \quad \forall A \in \mathfrak{L}\left(\ell^{2}\left(\mathbb{Z}_{+}\right) \otimes \mathbb{C}^{2}\right) \\ \rho_{0} & =\varrho\end{cases}
$$

as well as $\alpha(t)=g \mathbb{E}\left\langle Z_{t}(\xi), \sigma^{-} Z_{t}(\xi)\right\rangle$ and $\beta(t)=g \mathbb{E}\left\langle Z_{t}(\xi), a Z_{t}(\xi)\right\rangle$ (see, e.g., 37]). Therefore, $Z_{t}(\xi)$ is a strong $N^{p}$-solution of (44).

Let $Z_{t}(\xi)$ and $\widetilde{Z}_{t}(\xi)$ be strong $N^{p}$-solutions of (41) with initial datum $\xi$ belonging to $L_{N^{p}}^{2}\left(\mathbb{P}, \ell^{2}\left(\mathbb{Z}_{+}\right) \otimes \mathbb{C}^{2}\right)$. Then, $Z_{t}(\xi)$ is the strong $N^{p}$-solution of (16) with initial datum $\xi \in L_{N^{p}}^{2}\left(\mathbb{P}, \ell^{2}\left(\mathbb{Z}_{+}\right) \otimes \mathbb{C}^{2}\right), \alpha(t)=g \mathbb{E}\left\langle Z_{t}(\xi), \sigma^{-} Z_{t}(\xi)\right\rangle$ and $\beta(t)=$ $g \mathbb{E}\left\langle Z_{t}(\xi), a Z_{t}(\xi)\right\rangle$. Since $t \mapsto \mathbb{E}\left\langle Z_{t}(\xi), \sigma^{-} Z_{t}(\xi)\right\rangle$ and $t \mapsto \mathbb{E}\left\langle Z_{t}(\xi), a Z_{t}(\xi)\right\rangle$ are continuous functions, applying Theorems 4.1 and 4.2 , together with Theorem 3.2 of [37, we deduce that

$$
\mathbb{E}\left\langle Z_{t}(\xi), \sigma^{-} Z_{t}(\xi)\right\rangle, \quad \mathbb{E}\left\langle Z_{t}(\xi), a Z_{t}(\xi)\right\rangle, \quad \mathbb{E}\left\langle Z_{t}(\xi), \sigma^{3} Z_{t}(\xi)\right\rangle
$$

is a solution of (22) with initial condition $A(0)=\operatorname{tr}(a \varrho), S(0)=\operatorname{tr}\left(\sigma^{-} \varrho\right)$ and $D(0)=$ $\operatorname{tr}\left(\sigma^{3} \varrho\right)$. The same is true for $\widetilde{Z}_{t}(\xi)$ in place of $Z_{t}(\xi)$, and so Theorem 4.3 leads to $\mathbb{E}\left\langle Z_{t}(\xi), \sigma^{-} Z_{t}(\xi)\right\rangle=\mathbb{E}\left\langle\widetilde{Z}_{t}(\xi), \sigma^{-} \widetilde{Z}_{t}(\xi)\right\rangle$ and

$$
\mathbb{E}\left\langle Z_{t}(\xi), a Z_{t}(\xi)\right\rangle=\mathbb{E}\left\langle\widetilde{Z}_{t}(\xi), a \widetilde{Z}_{t}(\xi)\right\rangle
$$

for all $t \geq 0$. Now, the uniqueness of the strong $N^{p}$-solution of (16) implies $Z=\widetilde{Z}$.

On the other hand, suppose that $\left(\rho_{t}\right)_{t \geq 0}$ and $\left(\widetilde{\rho}_{t}\right)_{t \geq 0}$ are families of $N^{p}$-regular operators satisfying (54) such that $\rho_{0}=\widetilde{\rho}_{0}=\varrho$ and $t \mapsto \operatorname{tr}\left(a \rho_{t}\right), t \mapsto \operatorname{tr}\left(a \widetilde{\rho}_{t}\right)$ are continuous. Then, $\left(\rho_{t}\right)_{t \geq 0}$ is a $N^{p}$-weak solution to (14) with $\alpha(t)=g \operatorname{tr}\left(\sigma^{-} \rho_{t}\right)$ and $\beta(t)=$ $g \operatorname{tr}\left(a \rho_{t}\right)$, as well as $\left(\widetilde{\rho}_{t}\right)_{t \geq 0}$ is a $N^{p}$-weak solution to (14) with $\alpha(t)=g \operatorname{tr}\left(\sigma^{-} \widetilde{\rho}_{t}\right)$ and $\beta(t)=g \operatorname{tr}\left(a \widetilde{\rho}_{t}\right)$. Using Theorem 4.2 we get that $\left(\operatorname{tr}\left(a \rho_{t}\right), \operatorname{tr}\left(\sigma^{-} \rho_{t}\right), \operatorname{tr}\left(\sigma^{3} \rho_{t}\right)\right)$ and

$$
\left(\operatorname{tr}\left(a \widetilde{\rho}_{t}\right), \operatorname{tr}\left(\sigma^{-} \widetilde{\rho}_{t}\right), \operatorname{tr}\left(\sigma^{3} \widetilde{\rho}_{t}\right)\right)
$$


are solutions of (22) with initial condition $A(0)=\operatorname{tr}(a \varrho), S(0)=\operatorname{tr}\left(\sigma^{-} \varrho\right)$ and $D(0)=$ $\operatorname{tr}\left(\sigma^{3} \varrho\right)$. Since the solution of (22) is unique (see, e.g., Theorem 4.3), $\operatorname{tr}\left(a \rho_{t}\right)=$ $\operatorname{tr}\left(a \widetilde{\rho}_{t}\right), \operatorname{tr}\left(\sigma^{-} \rho_{t}\right)=\operatorname{tr}\left(\sigma^{-} \widetilde{\rho}_{t}\right)$ and $\operatorname{tr}\left(\sigma^{3} \rho_{t}\right)=\operatorname{tr}\left(\sigma^{3} \widetilde{\rho}_{t}\right)$. Therefore, $\left(\rho_{t}\right)_{t \geq 0}$ and $\left(\widetilde{\rho}_{t}\right)_{t \geq 0}$ are $N^{p}$-weak solution to (14) with the same $\alpha(t)$ and $\beta(t)$, and hence using Theorem 4.1 yields $\rho_{t}=\widetilde{\rho}_{t}$ for all $t \geq 0$.

\section{References}

[1] R. Alicki and K. Lendi. Quantum dynamical semigroups and applications, volume 717 of Lecture Notes in Physics. Springer, Berlin, second edition edition, 2007.

[2] A. Arnold and C. Sparber. Quantum dynamical semigroups for diffusion models with hartree interaction. Comm. Math. Phys., 251:179-207, 2004.

[3] A. Barchielli and M. Gregoratti. Quantum trajectories and measurements in continuous time: the diffusive case, volume 782 of Lecture Notes in Physics. Springer, Berlin, 2009.

[4] A. Barchielli and A. S. Holevo. Constructing quantum measurement processes via classical stochastic calculus. Stochastic Process. Appl., 58:293-317, 1995.

[5] V. P. Belavkin. Quantum branching processes and nonlinear dynamics of multiquantum systems. Soviet Math Dokl, 301:1348-1352, 1988.

[6] V. P. Belavkin. Multiquantum systems and point processes I. Generating functionals and nonlinear semigroups. Rep. Math. Phys., 28:57-90, 1989.

[7] H. P. Breuer, E. M. Laine, J. Piilo, and B. Vacchini. Colloquium: Non-markovian dynamics in open quantum systems. Rev. Mod. Phys., 88:021002, 2016.

[8] H.P. Breuer and F. Petruccione. The theory of open quantum systems. Oxford University Press, 2002.

[9] B. Bylicka, D. Chruscinski, and S. Maniscalco. Non-markovianity and reservoir memory of quantum channels: a quantum information theory perspective. Scientific Reports, 4:5720, 2014.

[10] A. M. Chebotarev, J. Garcia, and R. Quezada. On the Lindblad equation with unbounded variable coefficients. Math. Notes, 61:105-117, 1997.

[11] A. M. Chebotarev, J. Garcia, and R. Quezada. A priori estimates and existence theorems for the Lindblad equation with unbounded time-dependent coefficients, volume 1035, pages 44-65. Publ. Res. Inst. Math. Sci. Kokyuroku, 1998.

[12] A.M. Chebotarev. Necessary and sufficient conditions for conservativeness of dynamical semigroups. J. Sov. Math., 56:2697-2719, 1991.

[13] A.M. Chebotarev. Lectures on quantum probability. Sociedad Matemática Mexicana, México, 2000.

[14] A.M. Chebotarev and F. Fagnola. Sufficient conditions for conservativity of quantum dynamical semigroups. J. Funct. Anal., 118:131-153, 1993.

[15] A.M. Chebotarev and F. Fagnola. Sufficient conditions for conservativity of minimal quantum dynamical semigroups. J. Funct. Anal., 153:382-404, 1998. 
[16] D. Chruscinski and S. Maniscalco. On the degree of non-markovianity of quantum evolution. Phys. Rev. Lett., 112:120404, 2014.

[17] E.B. Davies. Quantum dynamical semigroups and the neutron diffusion equation. Rep. Math. Phys., 11:169-188, 1977.

[18] F. Fagnola. Quantum markov semigroups and quantum flows. Proyecciones, 18:1-144, 1999.

[19] F. Fagnola and C. M. Mora. Stochastic Schrödinger equations and applications to Ehrenfest-type theorems. ALEA, Lat. Am. J. Probab. Math. Stat., 10:191-223, 2013.

[20] F. Fagnola and C.M. Mora. On the relationship between a quantum Markov semigroup and its representation via linear stochastic Schrödinger equations. Indian J. Pure Ap. Mat., 46:399-414, 2015.

[21] A. C. Fowler, J. D. Gibbon, and M. McGuinness. The complex Lorenz equations. Physica D, 4:139-163, 1982.

[22] G. Friesecke and M. Koppen. On the Ehrenfest theorem of quantum mechanics. J. Math. Phys., 50:08210, 2009.

[23] G. Friesecke and B. Schmidt. A sharp version of Ehrenfest's theorem for general self-adjoint operators. Proc. R. Soc. A, 466:2137-2143, 2010.

[24] V. Gorini, A. Kossakowski, and E.C.G. Sudarshan. Completely positive semigroups of N-level systems. J. Math. Phys., 17:821-825, 1976.

[25] H. Haken. Light Vol. II: Laser light dynamics. North Holland, 1985.

[26] M. J. W. Hall, J. D. Cresser, L. Li, and E. Andersson. Canonical form of master equations and characterization of non-markovianity. Phys. Rev. A, 89:42120, 2014.

[27] K. Hepp and E. H. Lieb. Phase transitions in reservoir-driven open systems with applications to lasers and superconductors. Helv. Phys. Acta, 46:573-602, 1974.

[28] M.W. Hirsch, S. Smale, and R. L. Devaney. Differential equations, dynamical systems, and an introduction to chaos. Second edition. Elsevier, Amsterdam, 2013.

[29] T. Kato. Perturbation theory for linear operators. Corrected printing of the second edition. Springer, 1980.

[30] V.N. Kolokoltsov. Nonlinear Markov processes and kinetic equations. Cambridge University Press, 2010.

[31] G. Lindblad. On the generators of quantum dynamical semigroups. Commun. Math. Phys., 48:119-130, 1976.

[32] M. Merkli and G. P. Berman. Mean-field evolution of open quantum systems: an exactly solvable model. Proc. R. Soc. A, 468:3398-3412, 2012.

[33] C. M. Mora, J. Fernández, and R. Biscay. Numerical solution of stochastic master equations by exponential schemes. J. Comput. Phys., pages 28-48, 2018.

[34] C.M. Mora. Numerical simulation of stochastic evolution equations associated to quantum Markov semigroups. Math. Comp., 73:1393-1415, 2004. 
[35] C.M. Mora. Numerical solution of conservative finite-dimensional stochastic Schrödinger equations. Ann. Appl. Probab., 15:2144-2171, 2005.

[36] C.M. Mora. Heisenberg evolution of quantum observables represented by unbounded operators. J. Funct. Anal., 255:3249-3273, 2008.

[37] C.M. Mora. Regularity of solutions to quantum master equations: a stochastic approach. Ann. Probab., 41:1978-2012, 2013.

[38] C.M. Mora and R. Rebolledo. Regularity of solutions to linear stochastic Schrödinger equations. Infinite Dimens. Anal. Quantum Probab. Rel. Topics, 10:237-259, 2007.

[39] C.M. Mora and R. Rebolledo. Basic properties of non-linear stochastic Schrödinger equations driven by Brownian motions. Ann. Appl. Probab., 18:591$619,2008$.

[40] T. Mori. Exactness of the mean-field dynamics in optical cavity systems. J. Stat. Mech., 2013:P06005, 2013.

[41] C. Z. Ning and H. Haken. Detuned lasers and the complex Lorenz equations: Subcritical and supercritical Hopf bifurcations. Phys. Rev. A, 41:3826-3837, 1990.

[42] J. Ohtsubo. Semiconductor Lasers, volume 11 of Springer Series in Optical Sciences. Springer, Berlin, third edition, 2013.

[43] I.C. Percival. Quantum state diffusion. Cambridge University Press, 1998.

[44] C. Prévôt and M. Röckner. A concise course on stochastic partial differential equations, volume 1905 of Lecture Notes in Mathematics. Springer, Berlin, 2007.

[45] R. Schack, T. A. Brun, and I. C. Percival. Quantum state diffusion, localization and computation. J. Phys. A: Math. Gen., 28:5401-5413, 1995.

[46] T. Schulte-Herbrüggen, G. Dirr, and R. Zeier. Quantum systems theory viewed from Kossakowski-Lindblad Lie semigroups - and vice versa. Open Syst. Inform. Dynam., 24:1-25, 2017.

[47] H. Spohn. Kinetic equations from Hamiltonian dynamics: Markovian limits. Rev. Modern Phys., 52:569-615, 1980.

[48] G. H. M. van Tartwijk and G. P. Agrawal. Laser instabilities: a modern perspective. Prog. Quant. Electron., 22:43-122, 1998.

[49] H.M. Wiseman and G.J. Milburn. Quantum Measurement and Control. Cambridge University Press, 2009. 